\title{
Blowfly flight characteristics are shaped by environmental features and controlled by optic flow information
}

\author{
Roland Kern*, Norbert Boeddeker, Laura Dittmar and Martin Egelhaaf \\ Department of Neurobiology and Center of Excellence, Cognitive Interaction Technology, Bielefeld University, D-33501 Bielefeld, \\ Germany \\ *Author for correspondence (roland.kern@uni-bielefeld.de)
}

\begin{abstract}
SUMMARY
Blowfly flight consists of two main components, saccadic turns and intervals of mostly straight gaze direction, although, as a consequence of inertia, flight trajectories usually change direction smoothly. We investigated how flight behavior changes depending on the surroundings and how saccadic turns and intersaccadic translational movements might be controlled in arenas of different width with and without obstacles. Blowflies do not fly in straight trajectories, even when traversing straight flight arenas; rather, they fly in meandering trajectories. Flight speed and the amplitude of meanders increase with arena width. Although saccade duration is largely constant, peak angular velocity and succession into either direction are variable and depend on the visual surroundings. Saccade rate and amplitude also vary with arena layout and are correlated with the 'time-to-contact' to the arena wall. We provide evidence that both saccade and velocity control rely to a large extent on the intersaccadic optic flow generated in eye regions looking well in front of the fly, rather than in the lateral visual field, where the optic flow at least during forward flight tends to be strongest.
\end{abstract}

Key words: insect, saccade, obstacle avoidance, translation velocity, flight control, active vision.

Received 16 June 2011; Accepted 23 March 2012

\section{INTRODUCTION}

A distinguishing behavioral feature of many insects including flies is their saccadic flight style. It is characterized by periods where body orientation stays relatively constant and by brief saccadic turns where the animal reaches high rotation velocities (e.g. Boeddeker et al., 2005; Boeddeker et al., 2010; Braun et al., 2010; Braun et al., 2012; Collett and Land, 1975; Geurten et al., 2010; Mronz and Lehmann, 2008; Ribak and Swallow, 2007; Schilstra and van Hateren, 1999; Tammero and Dickinson, 2002a; Wagner, 1985; Wagner, 1986; Zeil, 1983; Zeil, 1986). Body saccades are accompanied by head saccades. Saccades of body and head differ in various aspects (Boeddeker et al., 2010; van Hateren and Schilstra, 1999), which can be essential for the nervous system to extract spatial information from the retinal image displacements (Kern et al., 2006). Saccadic turns are difficult to infer from the flight trajectories of large insects, such as blowflies or bees, which, probably as a consequence of inertia, change their overall direction smoothly (Boeddeker et al., 2005; Schilstra and van Hateren, 1999). In contrast, in the much smaller Drosophila, saccadic turns have been inferred on the basis of the flight trajectories, which look much more jerky than those of large insects (e.g. Tammero and Dickinson, 2002a; Mronz and Lehmann, 2008).

All changes in flight behavior directly affect the visual input of the animal, as movements of the animal lead to characteristic retinal image displacements. This optic flow (OF) yields cues about the animal's self-motion as well as the three-dimensional structure of the environment, and is a source of information for course control (for reviews, see Collett, 2002; Gibson, 1979; Koenderink, 1986; Lappe, 2000). Flight speed, for instance, depends on environmental clearance, as has been shown for bees (e.g. Baird et al., 2010; Portelli et al., 2011; Srinivasan et al., 1996). Yet it is not entirely clear how saccade amplitudes depend on the spatial layout of the environment during free flight. Saccade amplitude is highly variable (e.g. Boeddeker et al., 2010; Mronz and Lehmann, 2008; Schilstra and van Hateren, 1999; Tammero and Dickinson, 2002a; van Hateren and Schilstra, 1999), and depends to some degree on previously learnt information (see Hesselberg and Lehmann, 2009; Menzel, 2009) and the internal state of the animal (e.g. Budick and Dickinson, 2006; Cardé and Willis, 2008; McArthur and Dickman, 2011). For both freely flying and tethered Drosophila, saccades were concluded to be directed away from the eye experiencing image expansion, for example during obstacle approach (Bender and Dickinson, 2006; Budick et al., 2007; Reiser and Dickinson, 2010; Tammero and Dickinson, 2002a; Tammero and Dickinson, 2002b; Tammero et al., 2004). In contrast, collision avoidance of blowflies could be successfully accounted for by a behavioral model ('CyberFly') in which the initiation and direction of saccades is controlled through the output of a pair of wide-field neurons, each excited by front-toback motion in one visual hemifield (Lindemann et al., 2008).

In this study we challenge, by targeted modifications of the width and layout of the flight arenas, three OF-based mechanisms to predict the direction of saccades and compare these predictions with the blowflies' behavior. Two mechanisms use expansion OF fields according to studies in Drosophila (Tammero and Dickinson, 2002a; Reiser and Dickinson, 2010) and one mechanism is based on intersaccadic OF strength.

Not only saccade frequency and amplitude vary with the layout of the flight arena. Rather, we find that blowflies adjust their translation velocity depending on environmental clearance in a manner similar to that of honeybees (e.g. Baird et al., 2010; Portelli et al., 2011; Srinivasan et al., 1996). From the intersaccadic OF 
fields during intervals, we suggest eye regions that are potentially involved in the control of translation velocity.

\section{MATERIALS AND METHODS}

We recorded the behavior of 2- to 7-day-old female blowflies of the genus Lucilia taken from our laboratory colony.

\section{Setup}

We used two different types of flight tunnels, one with obstacles ('obstacle tunnel') and three straight tunnels of different width. All tunnels were covered with a Perspex lid and were illuminated from below by eighteen $50 \mathrm{~W}$ halogen lamps (luminance in the middle of the arena was $250 \mathrm{cdm}^{-2}$ ). Temperature was kept between 27 and $33^{\circ} \mathrm{C}$.

\section{Obstacle tunnel}

The walls and floor of this tunnel $(230 \times 40 \times 20.5 \mathrm{~cm}$, length $\times$ width $\times$ height; Fig. 1A) were covered with a red-and-white random square pattern (pixel size: $4 \times 4 \mathrm{~mm}$ ). This pattern exhibits good contrast to the fly and facilitates the detection of the animal on the images. Two Perspex obstacles $(0.6 \times 26 \times 20.5 \mathrm{~cm})$ were installed in the tunnel. The first obstacle was attached to the left wall perpendicularly to the tunnel's long axis $108 \mathrm{~cm}$ from the entrance, the second one was attached to the right wall $128 \mathrm{~cm}$ from the entrance. The first obstacle was covered with the same red-and-white random pattern lining the tunnel. For recording purposes, the pattern was printed on transparent plastic film sheets. The second obstacle carried a red random square pattern printed on standard white paper (pixel size: $17 \times 17 \mathrm{~mm}$ ).

\section{Straight tunnels}

The basic tunnel $(230 \times 36 \times 20.5 \mathrm{~cm})$ had adjustable sidewalls starting $0.8 \mathrm{~m}$ from the entrance (Fig. 1B). These allowed us to narrow the tunnel from 36 to 18 or $9 \mathrm{~cm}$. Movable flaps mounted at the beginning and the end of the sidewalls served as a guide railing. The walls and floor of the tunnel were covered with a red random square pattern (pixel size: $4 \times 4 \mathrm{~mm}$ ).

\section{Experimental procedure}

The flies' trajectories in the obstacle tunnel were filmed by two orthogonally arranged high-speed cameras, one above the tunnel and the other at its entrance ( 500 frames s$^{-1}$ sampling rate, $1024 \times 1024$ pixels; MotionPro, Redlake, San Diego, CA, USA). The filmed area was $40 \times 40 \mathrm{~cm}$ at the tunnel top and encompassed the two obstacles. In the straight tunnels, both cameras were used to film the flies from above and, thus, to enlarge the recording area $(70 \times 40 \mathrm{~cm})$. In preliminary experiments we used a third camera, running at 50 frames s$^{-1}$, that was located at the entrance of the arena to judge changes in flight altitude, and found that they were small compared with the changes in the flies' lateral position.

Flies were tested individually. If they did not fly within 2 min, they were removed from the arena and tested again later. In the narrow straight tunnel $(9 \mathrm{~cm})$, some flies were reluctant to fly or did not fly at all, limiting the total number of tested flies and flights per fly. With the exception of the narrow tunnel, flies stopped flying within the recording area only rarely. Only trajectories in the direction away from the entrance hole were stored for later analyses. We analyzed only complete passages without wall, bottom or roof contact of the fly. Such contact occurred only rarely, apart from the narrow tunnel condition.

\section{Data analysis}

The recorded video sequences were automatically analyzed with software developed in our workgroup (Lindemann et al., 2003). For
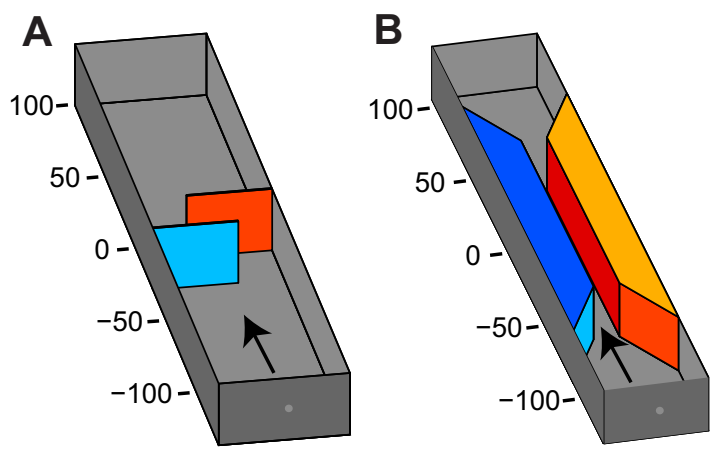

Fig. 1. Schematic diagrams of the flight arenas employed. (A) Tunnel $(230 \times 40 \times 20.5 \mathrm{~cm})$ with obstacles $(0.6 \times 26 \times 20.5 \mathrm{~cm})$ located 108 and $128 \mathrm{~cm}$ from the entrance. (B) Tunnel with adjustable sidewalls $(100 \times 1.5 \times 20.5 \mathrm{~cm})$ to narrow the tunnel from $36 \mathrm{~cm}$ to either 18 or $9 \mathrm{~cm}$, and flaps $(30 \times 1.5 \times 20.5 \mathrm{~cm})$ to guide flies to the bottleneck. Scaling is given in $\mathrm{cm}, 0 \mathrm{~cm}$ indicates centers of sections recorded by high-speed cameras (obstacle tunnel: $\pm 20 \mathrm{~cm}$; straight tunnels: $\pm 35 \mathrm{~cm}$ ). Arrows indicate flight direction. Colors are for illustration purposes only. Walls and floors of tunnels were covered with a red random square pattern (pixel size: $4 \times 4 \mathrm{~mm}$ ). The same holds for flaps in the straight tunnel and the first obstacle in the obstacle tunnel. The second obstacle carried a pattern of larger random squares $(17 \times 17 \mathrm{~mm})$. See Materials and methods for details.

each image, the coordinates of the fly's center of mass were detected and transformed into millimeter coordinates, using a Perspex calibration cube with markers on it. The top camera(s) (straight and obstacle tunnels) yielded the fly's $x$ - and $y$-coordinates, the back camera (obstacle tunnel only) the $x$ - and $z$-coordinates. By combining the top and back views, the time course of the fly's altitude in the obstacle tunnel was reconstructed (see Boeddeker et al., 2003). These data were used to confirm that the flight altitude remained relatively constant.

The limited spatial resolution of our video footage did not allow us to determine head yaw angles, but did allow the determination of body orientation (convention: $0 \mathrm{deg}=$ straight ahead along the longitudinal body axis; leftward orientations are assigned a positive angle, rightward ones a negative angle). All further data analysis was carried out using MATLAB (version 7.x, The MathWorks, Natick, MA, USA). $x$ - and $y$-coordinates as well as body orientation data were convolved with a Gaussian window (width $30 \mathrm{~ms}$, sigma $6 \mathrm{~ms}$ ) to reduce digitization jitter before calculation of velocity. If not stated otherwise, a turn was considered a saccade if the rotation velocity about the vertical body axis exceeded $400 \mathrm{deg} \mathrm{s}^{-1}$. Saccade duration was determined as its temporal width at half-maximum peak velocity. Saccade amplitude was approximated by the change in yaw angle between two crossings of a $200 \mathrm{degs}^{-1}$ velocity threshold. The flight distance was calculated as the sum of the flight sections traversed between two successive points of the flight trajectory projected into the $x-y$ plane.

\section{OF calculations}

The OF was calculated by an algorithm that determines - for every viewing direction ('sampling points') - the distance between the fly and the respective part of the flight arena (bottom, wall, obstacle, etc.) seen in this viewing direction (see Foley et al., 1996) and takes into account the next position change of the fly along the trajectory (Koenderink and van Doorn, 1987). The sample points were evenly spaced; their angular distance was either 2 or $5 \mathrm{deg}$ in azimuth and elevation as is specified below. The temporal resolution was $2 \mathrm{~ms}$. 
OF calculations are independent of contrast, illumination and texture of the walls.

We calculated the intersaccadic OF for different parts of the visual field (see Results) for intersaccadic intervals (ISIs) of at least $30 \mathrm{~ms}$. In the context of the control of translational velocity, OF analysis was based on the $30 \mathrm{~ms}$ interval prior to a saccade, i.e. before the yaw velocity exceeded $200 \mathrm{deg} \mathrm{s}^{-1}$ the next time. OF analysis in the context of saccade generation was limited to a $10 \mathrm{~ms}$ time window, ranging from 30 to $20 \mathrm{~ms}$ before the next saccade. All OF calculations are based on pure translatory intersaccadic movement, because intersaccadic head yaw shows virtually no rotational changes of yaw orientation during the ISI (Kern et al., 2006; van Hateren and Schilstra, 1999). Residual rotations were eliminated within the 10 or $30 \mathrm{~ms}$ interval by setting the yaw angle during the movement from point $\mathrm{A}(t=0 \mathrm{~ms})$ to point $\mathrm{B}(t=2 \mathrm{~ms})$ to the mean value of the two yaw angles at $A$ and $B$. Note that body yaw is not much different from head yaw orientation for most of the intersaccadic time (see van Hateren and Schilstra, 1999). The pitch angle - not resolved in the present study - was fixed at $20 \mathrm{deg}$ (head tilted upwards). The roll angle was set to $0 \mathrm{deg}$. These values correspond to the mean head angles during semi-free flights (Kern et al., 2006; van Hateren and Schilstra, 1999).

In addition to the flights filmed in this study, for $\mathrm{OF}$ analysis we used 10 head trajectories (each $3.45 \mathrm{~s}$ in length) recorded in a cubic arena (side length $40 \mathrm{~cm}$ ) by van Hateren and Schilstra (van Hateren and Schilstra, 1999). This was done to test proposed mechanisms for the control of saccades and translational velocity under a wider range of conditions. To facilitate comparison of the performance of the mechanisms across environments, we eliminated rotation velocities of the head by setting the yaw, the pitch and the roll angle to their mean values individually for each $2 \mathrm{~ms}$ interval of the ISIs. Therefore, only translational movements occur in all ISIs used in OF analysis. ISIs were defined as in our previous study (Kern et al., 2005).

\section{Predicting saccade direction and translation velocity from the OF}

From the $\mathrm{OF}$ in a $10 \mathrm{~ms}$ intersaccadic window (see above) we predicted the direction of the next saccade using three alternative methods. (1) Azimuth location of the focus of expansion (FOE): the FOE is defined as the location in the flow field from which all neighboring vectors point away. (2) Difference of expansioncongruency of $\mathrm{OF}$ in the left and right eye: while approaching an object, its retinal image expands. Expansion manifests itself in the $\mathrm{OF}$ as a radial pattern of velocity vectors around the focus of outflow. We calculated a measure of congruency of intersaccadic OF fields from such radial patterns separately for both eyes. The direction of the next saccade is predicted from the difference of the expansioncongruency measure obtained for the left and right eye. (3) Difference of OF strength: this measure is determined from the difference in OF strength in two selected areas, located symmetrically in the left and right visual field (for details see Results and the Fig. 12 legend).

To assess the visual field areas that might be relevant for controlling translation velocity, we compared intersaccadic OF strength in various parts of the visual field and determined the mean length of OF vectors within specific regions of the visual field $30 \mathrm{~ms}$ before the next saccade (details see legend of Fig. 5).

\section{RESULTS}

General flight characteristics

The flight trajectories of blowflies were seldom straight; they meandered (Fig. 2Bi) both in the flight tunnel with obstacles and in
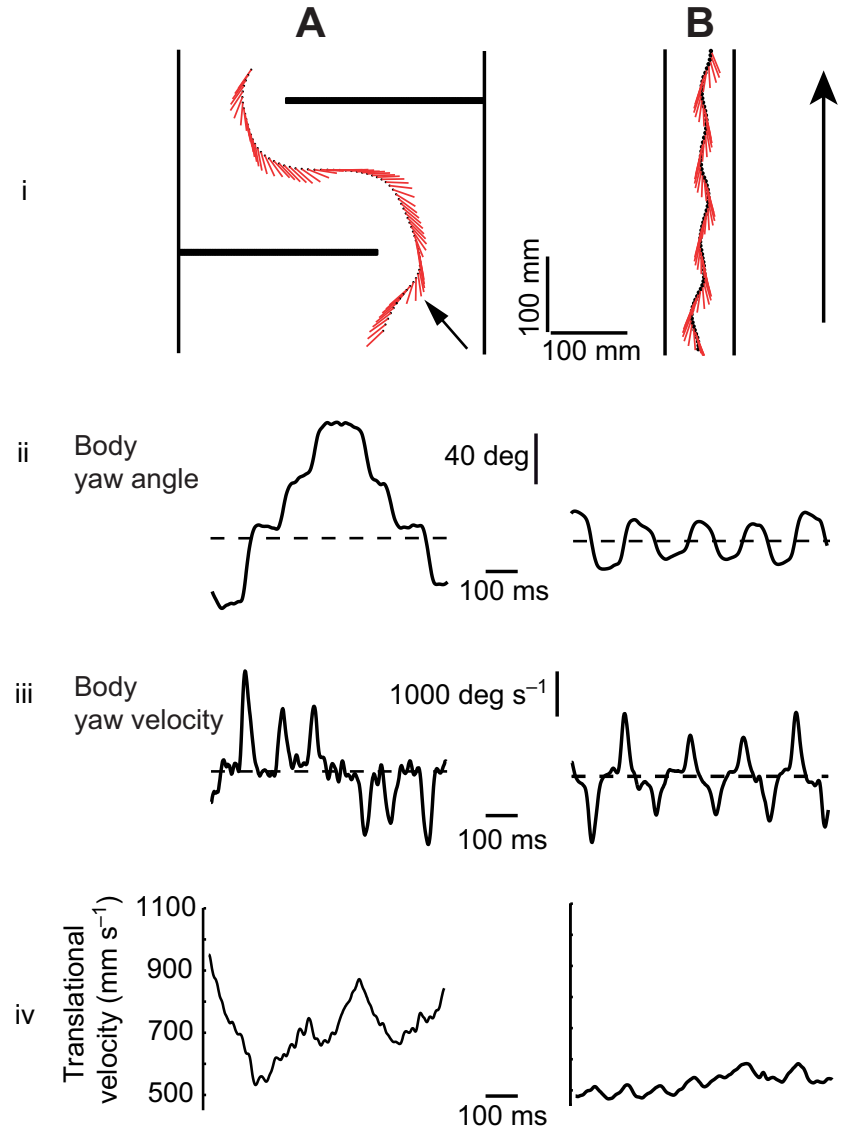

Fig. 2. Sample flights in the (A) obstacle and (B) narrow straight tunnels. (i) Reconstruction of the trajectory as seen from above. Position of the fly (black circles) and orientation of the body long axis (red lines) are projected every $10 \mathrm{~ms}$ in the horizontal plane. (ii-iv) Corresponding time courses of body orientation, angular velocity and translational velocity. In $\mathrm{Bi}$, only a fraction of the recorded trajectory (4/7) is displayed to match the scaling of Ai. Horizontal dashed lines in ii-iii indicate zero level. The arrow in $\mathrm{Ai}$ indicates a saccade. The arrow in $\mathrm{Bi}$ denotes the general flight direction in both arenas.

the straight tunnels, i.e. without any obvious need to turn (Fig. 2Bii,iii). Turns were accompanied by rapid changes in the longitudinal body axis of up to $60 \mathrm{deg}$ in less than $40 \mathrm{~ms}$ with velocities of up to $3000 \mathrm{deg} \mathrm{s}^{-1}$ (Fig. 2Ai,iii). These saccades were separated by periods of much smaller turning (Fig. 2Aii). In the straight flight tunnels, saccade direction switched in a rather regular fashion. The saccadic turns were not immediately reflected in the flight trajectories (see also Schilstra and van Hateren, 1999), which showed only much smoother changes in direction that were not necessarily concurrent with the saccades (Fig. 2Ai). In our flight arenas, translational velocities ranged between 0.5 and $2 \mathrm{~m} \mathrm{~s}^{-1}$ and varied during the flights at a slower time scale than the rotational velocities (compare Fig. 2Aiv,Biv with 2Aiii,Biii).

Most flies used a relatively narrow $\mathrm{S}$-shaped flight corridor when negotiating the obstacle arena (Fig. 3A) and most of the trajectories ran close to the tunnel midline in the straight tunnels (Fig. 3B-D). Flies flew most frequently in central regions of the flight tunnels. This behavior is similar to the centering response in bees (Dyhr and Higgins, 2010; Kirchner and Srinivasan, 1989; Serres et al., 2008; Srinivasan et al., 1991), which is thought to be controlled by the OF in the lateral visual field (Srinivasan et al., 1991). 

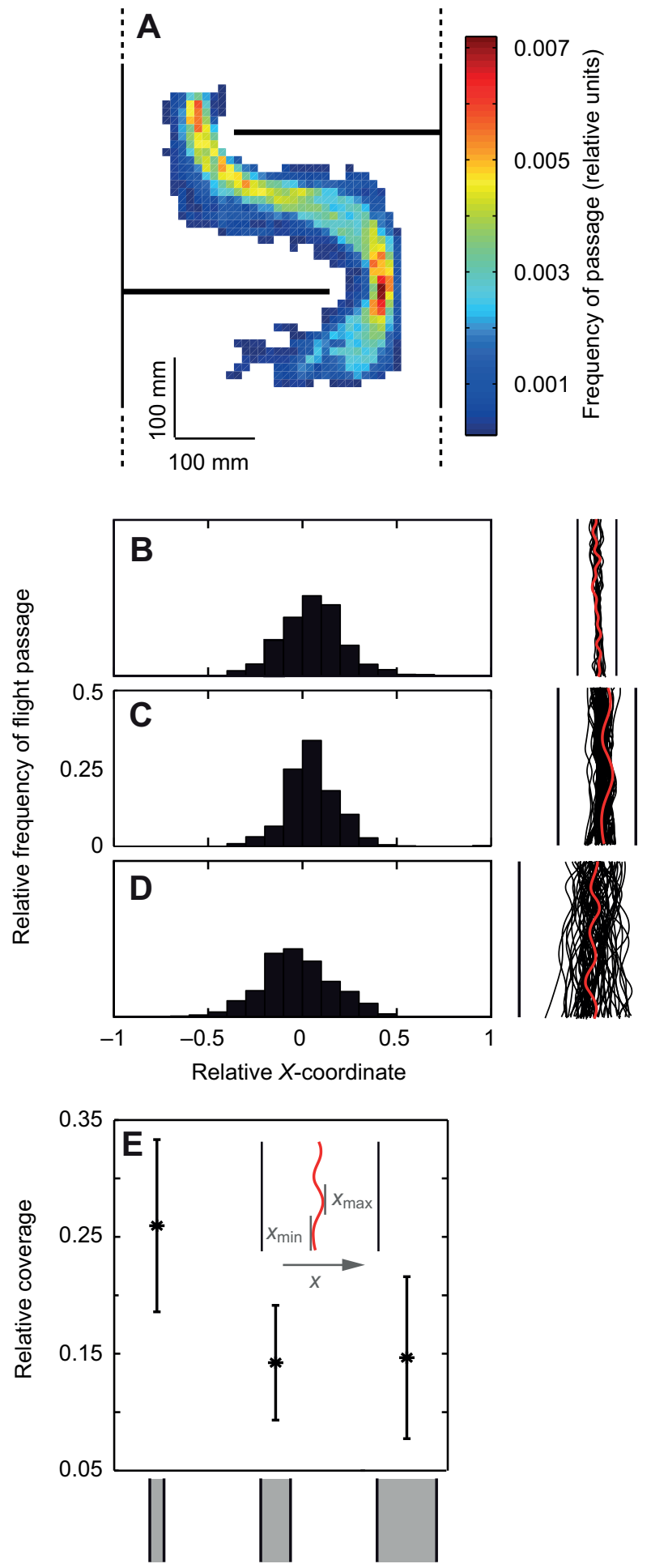

In the straight tunnels, the trajectories covered slightly less than $50 \%$ of the tunnel width (Fig. 3B-D). The frequency distributions of presence of the flies within axial strips of each flight tunnel normalized to the tunnel width are rather similar for all tunnels, i.e. the minimum distance to the walls seems to be controlled not in absolute but in relative terms. Single trajectories usually covered a smaller area of the tunnel than the entire population of trajectories (Fig. 3E, insets 3B-E). This finding implicates that single flies do not always center perfectly in the tunnels. They thus tolerate to some
Fig. 3. (A) Spatial probability distribution of flight passages in the obstacle arena. The arena was virtually overlaid by a square grid of $1 \times 1 \mathrm{~cm}$ elements. When a fly passed a given grid element, a data point was assigned to it irrespective of passage time. The relative frequencies of data points in every grid element for all flights of this fly were then calculated. These frequencies were averaged over all flies and plotted. The probability values of all grid elements sum to 1 ( $N=14$ flies, $n=115$ trajectories). (B-D) Relative frequencies of flight passage in the straight tunnels of different width. Each bar corresponds to an axial strip of a relative width of $5 \%$ of the tunnel diameter. Populations of trajectories are shown in insets (black lines); examples are highlighted in red. (E) Fraction of tunnel width ( \pm s.d.) utilized during single flights in the narrow $(N=5, n=21)$, medium $(N=11$, $n=61)$ and wide $(N=11, n=62)$ straight tunnels ( $x$-axis). Inset: $X_{\min }$ and $X_{\max }$, minimum and maximum coordinates of a trajectory along the tunnel cross axis $(X)$ used to calculate relative coverage.

extent imbalances of OF on the eyes. Flight trajectories of honeybees are also shifted towards either sidewall of the tunnel if their goal at the end of the tunnel is off-center (Serres et al., 2008), and flies with one eye covered tend to fly closer to the wall on the side of the open eye (R.K. and M.E., unpublished data).

\section{Dependence of translational velocity on the environment}

Does the fly's translational velocity depend on the structure of the environment? To assess to what extent the fly's translational (twodimensional) velocity depends on the environment, we modified the surroundings by changing the width and spatial layout of the flight arenas. In straight tunnels, translational velocity increased with increasing width (Fig. 4A). Mean translational velocities for nine out of 11 individual flies were larger in the wide than in the medium tunnel (Wilcoxon signed rank test, $P=0.0186$; Fig. 4B). Likewise, for the five flies that could be tested in all three straight tunnels, the mean translational velocities in the narrow tunnel were lowest (data not shown). Individual flies seem to have a preferred speed range in a given flight tunnel that could differ by more than a factor of two between individual flies (Fig. 4B). The mean preferred translational velocities varied to a larger extent between individual flies than within a given fly (Fig. 4B).

In the obstacle tunnel (maximum width $40 \mathrm{~cm}$ ), translational velocity changed not only from trajectory to trajectory but also at different locations along a trajectory (Fig. 4C). On average, it fell between the mean velocities in the narrow (width $9 \mathrm{~cm}$ ) and the medium tunnels (width $18 \mathrm{~cm}$; Fig. 4A). At first sight, this result appears to be in conflict with our finding that in the straight tunnels translational velocity increases with tunnel width. However, the constrictions in the flight corridor resulting from the obstacles (Fig. 4A) might lead to a decrease in flight velocity. In addition, fast turns, which were more frequent in the obstacle than in the straight tunnels, led to reductions in forward velocity (see below). Flight speed thus is expected to be smaller in the obstacle tunnel than in a straight tunnel of equal width.

\section{The role of OF in translational control}

Flight speeds in the straight tunnels increased with tunnel width. Thus, flight speed might be regulated by a mechanism that keeps the intersaccadic OF constant, as the translational OF depends on the distance to environmental structures. This has been concluded for bees (see Baird et al., 2010; Srinivasan et al., 1996). However, it is not clear a priori which eye regions are used for speed control. Therefore, we identified those eye regions in which the OF varied least (i.e. smallest standard deviation) during the last $30 \mathrm{~ms}$ of the ISI across a large number of different conditions (Fig. 5A-E). Fifteen 

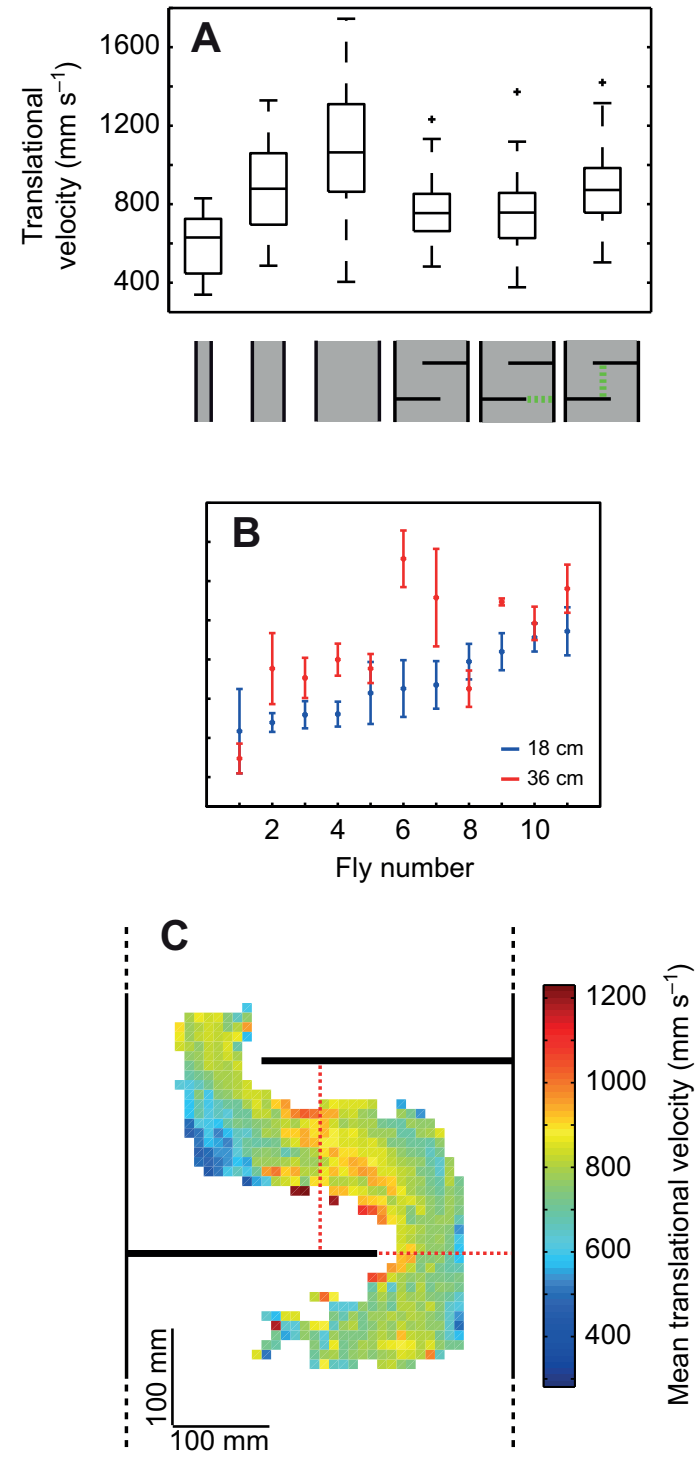

Fig. 4. (A) Median two-dimensional translational velocity in tunnels of different width in the entire obstacle arena or sections of it (indicated in green) [left to right: tunnel width $9 \mathrm{~cm}(N=5, n=21) ; 18 \mathrm{~cm}(N=11, n=61)$; $36 \mathrm{~cm}(N=11, n=62)$; entire obstacle arena $(N=14, n=115)$; level of first bottleneck (width $14 \mathrm{~cm}$ ); and level central axis (width $20 \mathrm{~cm}$ )]. The upper and lower margins of the boxes indicate the 75th and 25th percentiles, respectively, the whiskers the most extreme values not defined as outliers. Values larger (smaller) than the 75th (25th) percentile by more than 1.5 times the interquartile range are defined as outliers (+). (B) Mean ( \pm s.d.) translational velocity of 11 flies in the medium (blue) and wide (red) straight tunnels, in ascending order with respect to flight speed in the medium tunnel. (C) Spatial distribution (grid: $1 \times 1 \mathrm{~cm}$ ) of mean translational velocities in the obstacle tunnel.

regions of partly differing angular horizontal extent were located symmetrically with respect to the frontal midline. These always had a vertical extent of $40 \mathrm{deg}$ and were analyzed for five different elevations (Fig. 5A-E). The uppermost region was centered about the horizon (Fig. 5A); the lowest was centered 40 deg below the horizon (Fig. 5E). In addition to our flight tunnel data, we also included data from a previous study (van Hateren and Schilstra, 1999) because here the flight trajectories were more variable with respect to flight direction and height.
OF strengths, i.e. angular retinal velocities, varied least (in Fig. 5, blue shading) across the five different flight arenas for intermediate retinal elevations (Fig. 5B,C). Relatively large differences (yellow shading) between OF strength in the different arenas occurred independent of azimuthal size and location for regions that covered more ventral areas of the visual field (Fig. 5E). The biggest variation (red shading) across OF strength was found for eye regions close to the horizon that include the lateral eye (Fig. 5A). For example, the OF strength amounts to $532 \pm 166 \mathrm{degs} \mathrm{s}^{-1}$ in the narrow tunnel, $417 \pm 127 \mathrm{degs}^{-1}$ in the medium tunnel and $246 \pm 99 \mathrm{deg} \mathrm{s}^{-1}$ in the wide tunnel if the equatorial lateral visual field between azimuthal values of 60 and $90 \mathrm{deg}$ is considered. This large difference suggests that lateral eye regions are not used for velocity control. However, the average intersaccadic OF strength within a bilateral pair of $30 \mathrm{deg}$ wide frontolateral regions at an elevation of $20 \mathrm{deg}$ below the horizon (one of three regions colored dark blue in Fig. 5C) was close to $100 \mathrm{degs}^{-1}$ across flight arenas (Fig. 5F). The retinal velocities were only moderately larger (up to $140 \mathrm{deg} \mathrm{s}^{-1}$ ) for the other two frontolateral regions shown in dark blue in Fig. 5C.

Hence, a translational velocity controller that adjusts the OF to a pre-set value is likely to use OF from frontolateral and moderately ventral eye regions. If flight altitude was $10 \mathrm{~cm}$ above ground, as judged from our behavioral data and assumed for our OF calculations, features on the ground considered for flight speed control would have a minimal distance of approximately $12 \mathrm{~cm}$.

\section{Environmental dependence of saccade characteristics and saccade number}

Turning direction and velocities were not distributed homogeneously along the flight paths (shown in Fig. 6A for the obstacle tunnel). Rather, left turns prevailed before and during passage of the first obstacle, whereas right turns dominated the area before clearing the second obstacle.

The amplitudes of saccades, i.e. turns with peak velocities exceeding $400 \mathrm{deg} \mathrm{s}^{-1}$, were variable; most of them were smaller than $50 \mathrm{deg}$ in all flight arenas (Fig. 6B,C). Only in the obstacle tunnel were saccades with larger amplitudes frequent (compare Fig. 6B and 6C); here the median saccade amplitude was largest ( $28 \mathrm{deg}$ ) as compared with 16, 17 and $23 \mathrm{deg}$ in the narrow, medium and wide straight tunnels, respectively. Saccade amplitudes tended to be bigger when the fly approached the arena wall closely and, thus, with lower time-to-contact (Fig. 6D).

Saccade amplitudes were linearly related to saccade peak velocities (Fig. 6E), whereas saccade duration was rather constant for saccades larger than $20 \mathrm{deg}$ (Fig. 6F). The under-representation of small saccades may be the consequence of the saccade detection threshold employed. These characteristics do not depend much on the environment, as they resemble those published by Schilstra and van Hateren (Schilstra and van Hateren, 1999) for body saccades in free-flying Calliphora in a geometrically different environment. Also, the saccades of freely flying Drosophila (e.g. Mronz and Lehmann, 2008; Tammero and Dickinson, 2002a) and those elicited by visual expansion in magnetically tethered Drosophila [fig. 3 in Bender and Dickinson (Bender and Dickinson, 2006)] share characteristics with those measured in freely flying calliphorids [present study and Schilstra and van Hateren (Schilstra and van Hateren, 1999)]. Nevertheless, there are species-specific as well as methodologically grounded differences [see Bender and Dickinson (Bender and Dickinson, 2006) for a thorough discussion of the differences between saccades of tethered and freely flying Drosophila]. 

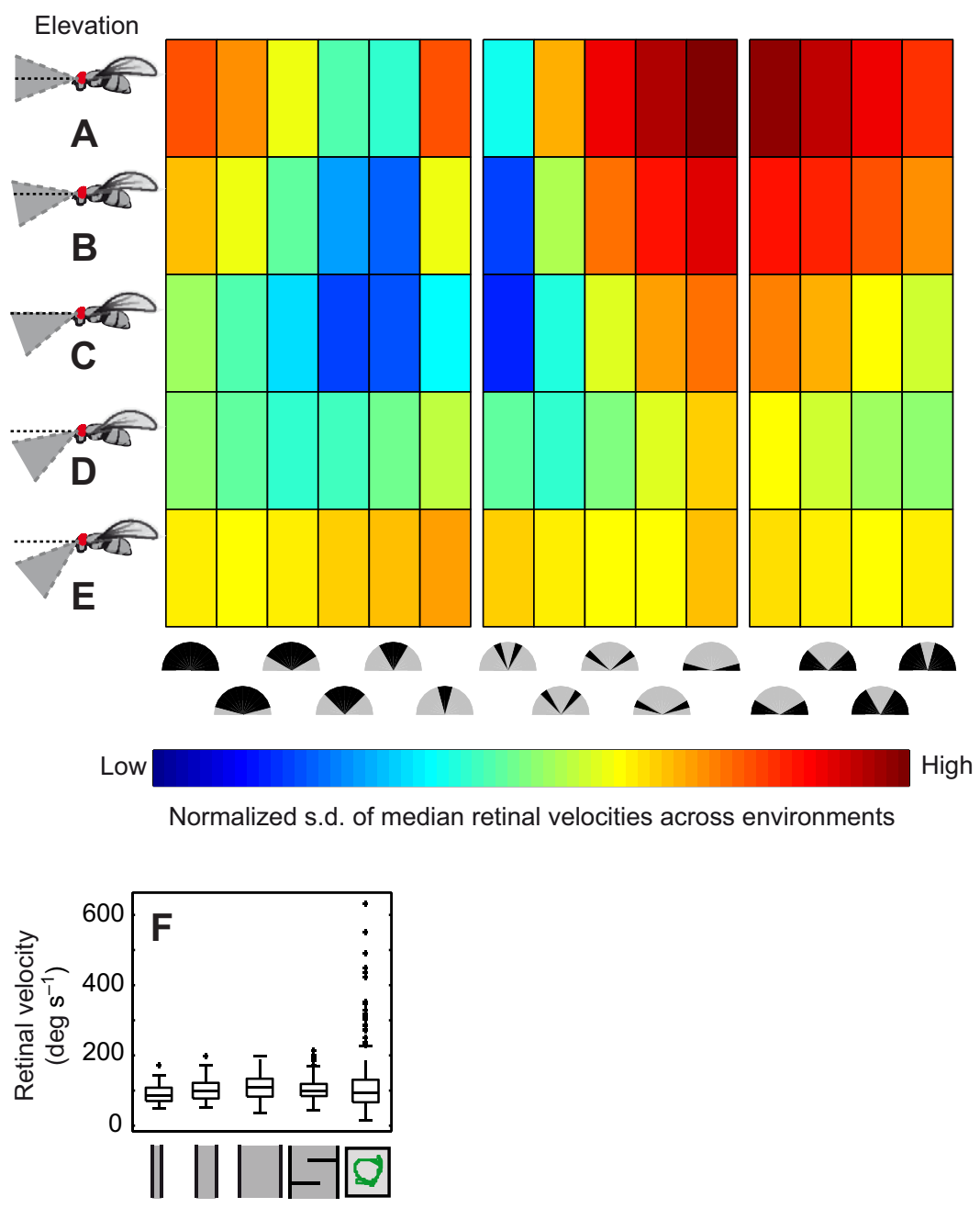

The geometry of the environment has an influence on saccade frequency that, on average, is approximately twice as large in the obstacle tunnel as in the straight tunnels (Fig. 7). Saccade rate increased via shortening the ISIs, whereas saccade duration was kept rather constant (not shown). If the OF experienced by the fly modulates saccade frequency, we expect - in a given environment - an influence of flight velocity on saccade number. However, saccade frequency did not depend consistently on flight speed in our flight arenas (Fig. 7). We obtain qualitatively the same results with saccade thresholds lower than the $400 \mathrm{deg} \mathrm{s}^{-1}$ used here.

Because in Drosophila the OF corresponding to an expanding object has been shown to trigger collision avoidance maneuvers, the likelihood and the size of saccades increases when the fly is heading towards a nearby obstacle (Tammero and Dickinson, 2002b). We therefore determined for all four flight arenas and the cubic arena (van Hateren and Schilstra, 1999) the ISI duration as a function of the time-to-contact to the arena wall $30 \mathrm{~ms}$ prior the next saccade (Fig. 8). The duration of an ISI is an indicator of how early the fly generates a saccade after a section of straight flight. The ISIs tended to become smaller as the time-to-contact to the arena wall decreased. This finding helps to understand the high mean saccade frequency in the obstacle tunnel, because here the time-to-contact, on average, is smaller than in the straight tunnels.

\section{Saccade localization and obstacle clearance}

Saccades occurred everywhere along the flight corridor of the obstacle tunnel (Fig. 3A) without obvious differences for the
Fig. 5. (A-E) Standard deviation of the medians of mean optic flow velocities in five different flight arenas as exemplified in F. Each standard deviation was normalized to the mean of the five respective median velocities. Calculations were performed for 75 (five rows $\times 15$ columns) regions of the visual field. Regions were centered about five elevations $(A-E)$ with respect to the external world. Negative values indicate centers below horizon $(0 \mathrm{deg})$, indicated by the horizontal dotted line in the fly graphics: $(A) 0$ deg, $(B)$ $-10 \mathrm{deg},(\mathrm{C})-20 \mathrm{deg}$, (D) $-30 \mathrm{deg}$ and (E) $-40 \mathrm{deg}$. In azimuth, 15 regions of different width, each symmetrically arranged about the frontal midline, were tested (see $x$-axis pictograms). These were, left to right (in deg): (1) 90 to -90 ; (2) 75 to -75 ; (3) 60 to -60 ; (4) 45 to -45 ; (5) 30 to -30 ; (6) 15 to -15 ; (7) 30 to 15 plus -30 to -15 ; (8) 45 to 30 plus -45 to -30 ; (9) 60 to 45 plus -60 to -45 ; (10) 75 to 60 plus -75 to -60 ; (11) 90 to 75 plus -90 to -75 ; (12) 90 to 60 plus -90 to -60 ; (13) 90 to 45 plus -90 to -45 ; (14) 90 to 30 plus -90 to -30 ; and (15) 90 to 15 plus -90 to -15 . (F) Median of mean retinal image velocities within intersaccadic intervals (ISIs) experienced in an example eye region for trajectories in the three tunnels of different width ( $9 \mathrm{~cm}: N=5, n=21,99$ ISIs; $18 \mathrm{~cm}: N=11, n=61,169$ ISIs; $36 \mathrm{~cm}$ : $N=11, \mathrm{n}=62,199$ ISIs), the obstacle tunnel ( $N=14$, $n=115,535$ ISIs) and in the cubic arena employed by van Hateren and Schilstra (van Hateren and Schilstra, 1999) ( $N=5, n=10,265$ ISIs) (see $x$-axis). Optic flow (sampling raster: $5 \times 5 \mathrm{deg}$ ) was always calculated for the $30 \mathrm{~ms}$ section of the ISI just before the saccade. The example region of 40 deg vertical extent was centered in elevation at -20 deg with respect to the horizon; azimuthal size was $60 \mathrm{deg}$, centered about the frontal midline (see $\mathrm{C}$, fifth column from left). different amplitude classes (Fig. 9A-D). Although a minimum of two saccades would suffice to fly around the obstacles, i.e. one to clear each obstacle, this 'minimal strategy' was not employed by blowflies in the present study. Most flights contained between four and eleven saccades (Fig. 9E). Even after traversing the obstacle tunnel several times, flies did not consistently use less saccades in later flights (flight numbers 6-9) as compared with their early (1-4) flights (Wilcoxon signed rank test, $P=0.4453, N=10$ ).

Are saccade amplitudes related to the total number of saccades used to clear the obstacles? In nearly $50 \%$ of flights we detected two saccades right to the edge of the first obstacle (rectangle in Fig. 6A). Flights with one or three saccades each occurred approximately $20 \%$ of the time; flights with four and five saccades were rare (Fig. 10A). The distributions of saccade amplitudes for single saccades, doublets or triplets overlapped substantially, with medians amounting to 31,37 and $36 \mathrm{deg}$, respectively (Fig. 10B).

\section{Saccade amplitude and translational velocity}

Saccadic turns seem to go along with changes in translational velocity (Fig. 2), and as the widest range of saccadic amplitudes was observed during flights in the obstacle arena, these data are used to quantify these changes. Similar results were obtained for the straight tunnels (data not shown).

For saccades exceeding $40 \mathrm{deg}$, translational velocity, in particular its forward component, was reduced considerably with a peak at approximately $50 \mathrm{~ms}$ after onset of the saccade (Fig. 11A,B). Thus, blowflies do not decelerate before the turn (as a car driver would), 

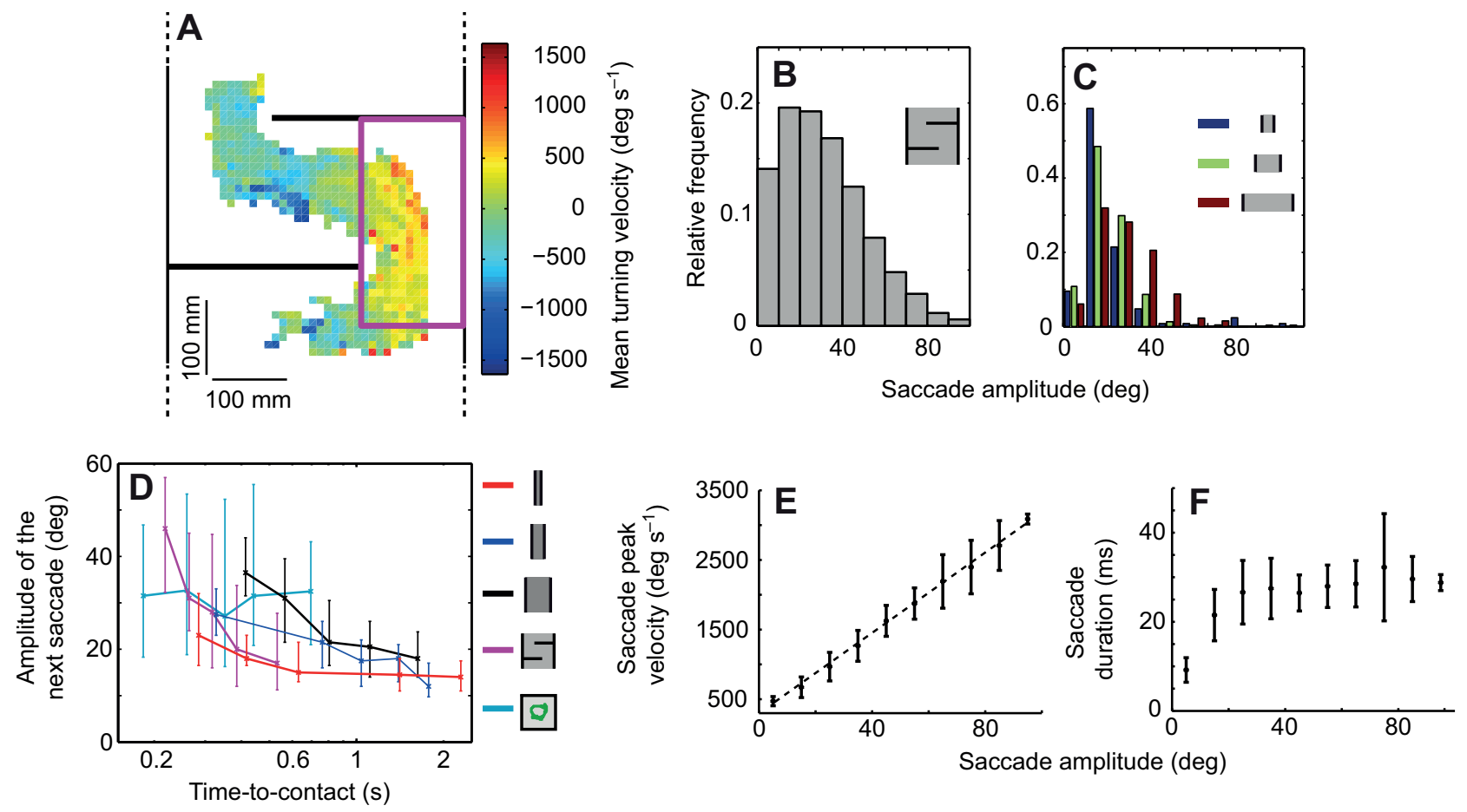

Fig. 6. Characteristics of turning. (A) Spatial distribution (grid: $1 \times 1 \mathrm{~cm})$ of mean turning velocity $(N=14, n=115)$. The magenta rectangle denotes the area for detection of saccades attributed to obstacle clearance. (B,C) Relative frequency of saccades within amplitude classes in the obstacle (B; $N=14, n=873)$ and three straight tunnels (C; narrow: $N=5, n=126$; medium: $N=11, n=231$; wide: $N=11, n=263$ ). (D) Amplitude ( \pm s.d.) of saccade versus time-to-contact in the preceding ISI for flight arenas of different width, the obstacle tunnel and the cubic arena (van Hateren and Schilstra, 1999) (see insets). Time-to-contact was calculated from flight velocity and the distance to environmental structures in flight direction of the animal $30 \mathrm{~ms}$ before the next saccade. Time-to-contact was binned so that every class contained approximately the same number of entries for a given flight arena (number of entries: narrow: $20 \pm 1$; medium: $34 \pm 1$; wide: $40 \pm 1$; obstacle: 107 ; cubic arena: 53 ). (E) Mean ( \pm s.d.) saccade peak velocity versus mean saccade amplitude within classes of 10 deg width. (F) Mean ( \pm s.d.) saccade duration versus mean saccade amplitude within classes of 10 deg width.

but only during and after it. In parallel, the sideways velocity component may increase considerably, implying that after saccades flies continue to move into their pre-saccadic flight direction for considerable portions of the ISI. Such sideways velocities were seen not only after large saccades, but also after small saccades. They increased with saccade amplitude (Fig.11C). The appearance of sideways movement after saccades of low amplitude hints at a function of saccades in the context of distance information retrieval from $\mathrm{OF}$ in the straight tunnels as well as in the obstacle tunnel (see Discussion).

\section{The role of OF in controlling saccade direction}

How might saccades be controlled by OF? In previous studies on flies, OF has been proposed to play a decisive role in determining the timing and direction of saccades (e.g. Dickinson, 2005; Lindemann et al., 2008; Mronz and Lehmann, 2008). Therefore, we determined three measures based on the intersaccadic OF that might be relevant in controlling saccade direction. Fig. 12 exemplifies flow fields for two time steps along a sample trajectory in the obstacle tunnel; Fig. 13 then shows the averaged data obtained for all environments.

\section{Prediction of saccade direction based on the difference of expansion congruency of intersaccadic OF}

This measure is related to the procedure employed by Tammero and Dickinson (Tammero and Dickinson, 2002a). Assuming centers of expansion at $46 \mathrm{deg}$ to the left and right of the frontal midline, the horizontal OF was summed separately in four areas (Fig. 12A, top). The vertical extent of each area was $40 \mathrm{deg}$, centered about the horizon. The vertical OF was summed separately in four areas located as shown in Fig. 12A (bottom). Then, horizontal expansions were calculated as the difference of the summed signed (see inset top right) horizontal flow components $(\mathrm{H}$, see inset) between areas 1 and 2 (left) as well as areas 3 and 4 (right). Vertical expansions were calculated as the difference of the summed signed vertical flow components (V, see inset) between areas 5 and 7 (left) as well as areas 6 and 8 (right). Horizontal and vertical expansions on either side were summed to yield the left and right total expansion. Saccades were predicted to be directed to the side corresponding to the smaller of these two numbers. In addition to expansion centers assumed at $46 \mathrm{deg}$ to the left and right, we employed the same procedure for expansion centers at $\pm 30 \mathrm{deg}$. Areas $1-4$ were of equal size, i.e. we omitted the most lateral parts of the visual field. According to the rule sketched for the example flow field in Fig. 12A, the next saccade is predicted correctly to be leftwards, independent of the location of the assumed expansion centers. Saccade number two (Fig. 12B), however, is predicted to be leftwards for expansion centers at $\pm 30 \mathrm{deg}$, but to be rightwards for expansion centers at $\pm 46 \mathrm{deg}$. Taking all flight data together (Fig. 13B), the predictions are correct and are well above chance level for all environments, and even exceed $80 \%$ in two cases for the centers of image expansion at $\pm 30 \mathrm{deg}$. In contrast, if the centers of expansion are located at $\pm 46 \mathrm{deg}$, the percentage of correct predictions is close to chance level for three environments. Only for the cubic arena is the level of correct predictions higher. We conclude that the saccade direction can be well predicted based on the OF expansion congruency across 

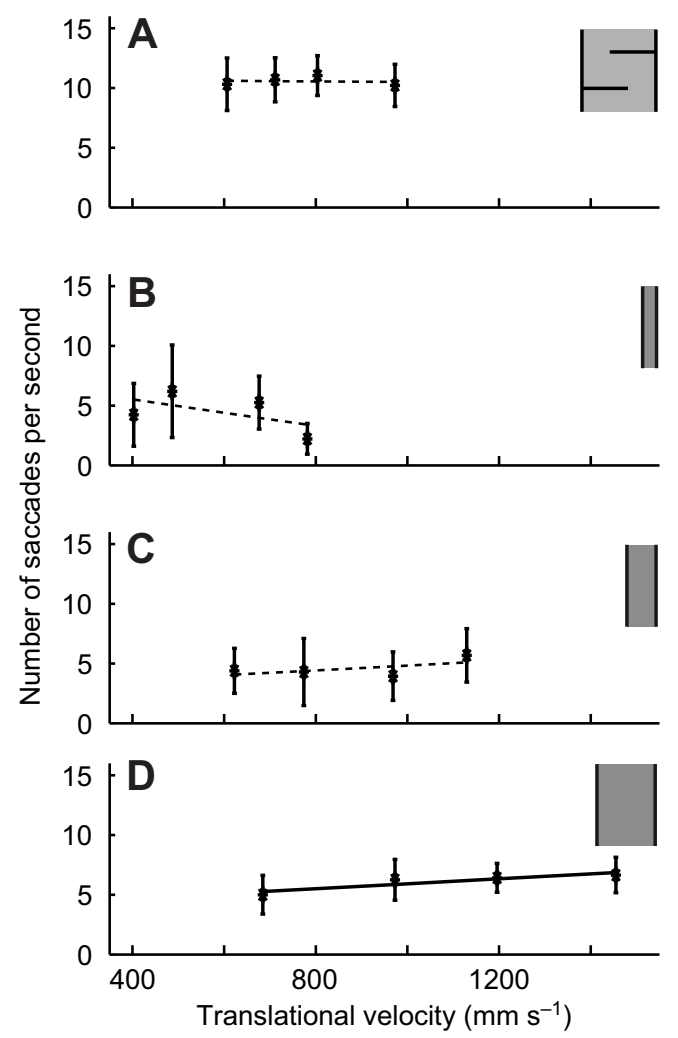

Fig. 7. Dependence of mean ( \pm s.d.) number of saccades per second in the (A) obstacle and $(B-D)$ straight tunnels on the mean translation velocity along the respective trajectory (A: $N=14, n=115 ; \mathrm{B}: N=5, n=21 ; \mathrm{C}: N=11$, $n=61$; $\mathrm{D}: N=11, n=62$ ). Data points were binned into four velocity classes such that every class contains about the same number of entries $(A: 29 \pm 1$; B: $6 \pm 1$; C: $16 \pm 1$; D: $16 \pm 1$.

environments, if the center of expansion is sufficiently frontal in the visual field.

\section{Prediction of saccade direction based on the difference of intersaccadic OF strength}

OF strength was approximated by the lengths of the flow vectors, summed separately within six equally sized areas, covering in azimuth the complete visual field from $90 \mathrm{deg}$ left to $90 \mathrm{deg}$ right (Fig. 12B). The vertical extent of each area was $40 \mathrm{deg}$, centered about the horizon. Saccades were directed towards the side of the area experiencing the lower OF strength. From the example flow field shown in Fig. 12A, the next saccade is correctly predicted according to this rule to be directed leftwards, independent of the pair of areas compared with each other. Saccade number two (Fig. 12B), however, is predicted to be leftwards only if the frontolateral areas of the visual field are exploited. From the difference between total OF in either the lateral or frontal areas, the prediction is rightwards. Taking all flight data together, predictions based on the total intersaccadic OF in the lateral eye regions are poor for all but the cubic arena (green areas/markers in Fig. 13C). The OF in only the frontal eye regions (red areas/markers in Fig. 13C) results in a better performance, especially in the straight tunnels, where it even exceeds a level of $80 \%$ for the two narrow tunnels; however, only chance performance was reached for the obstacle tunnel. The best overall performance was achieved with correct predictions well above chance level for all tested environments when we took the intersaccadic OF in frontolateral

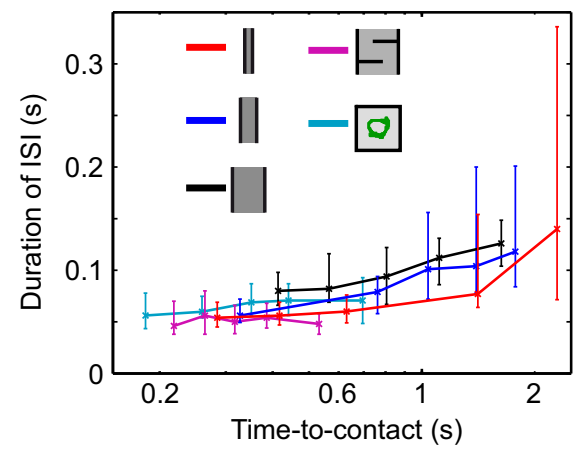

Fig. 8. Mean ( \pm s.d.) duration of ISIs versus time-to-contact for data from flight arenas of different width, the obstacle tunnel and the cubic arena (van Hateren and Schilstra, 1999). Time-to-contact was calculated from flight velocity and distance to environmental structures in the flight direction $30 \mathrm{~ms}$ before the next saccade. Time-to-contact was binned so that every class contained approximately the same number of entries for a given flight arena (number of entries: narrow: $20 \pm 1$; medium: $34 \pm 1$; wide: $40 \pm 1$; obstacle: 107; cubic arena: 53).

areas into account (blue areas/markers in Fig. 13C). Note that we obtained qualitatively the same results if we took only the signed horizontal components of the OF vectors rather than the entire vector length into account.

Prediction of saccade direction based on FOE location When characterizing the turning responses elicited by expansion flow fields, especially in experiments on tethered flies, it is frequently assumed that the FOE coincides with the location of the most pronounced image expansion (e.g. Reiser and Dickinson, 2010). We therefore also attempted to predict saccade direction from the azimuthal FOE location. A saccade away from the side of the FOE in the preceding ISI was regarded as predicted correctly. Predictions are correct significantly above chance level only for the obstacle tunnel (Fig. 13A). For the cubic arena (van Hateren and Schilstra, 1999), prediction performance is close to chance level, whereas for the straight tunnels predictions based on the FOE bearing are significantly worse than chance level. Hence, the prediction of saccade direction based on FOE location does not account for the experimental data.

These results suggest that exploiting the expansion congruency of intersaccadic OF with a center of expansion at $30 \mathrm{deg}$ or the overall intersaccadic OF across corresponding frontolateral regions of both eyes is a much more robust, though far from perfect, predictor of the direction of saccades than is the location of the FOE. On the whole, the expansion congruency of intersaccadic OF with a center of expansion at $30 \mathrm{deg}$ is the best predictor of saccade direction under the variety of environmental conditions tested here. In any case, if the prediction mechanism also takes into account the lateral areas of the visual field, the performance decreases for most tested environments.

\section{DISCUSSION}

Blowflies modify the frequency, direction and amplitude of their saccadic turns as well as their translational flight velocity with respect to the shape of the environment. We provide evidence for the hypothesis that the control of both saccades and translational velocity rely to a large extent on the intersaccadic OF generated during self-motion of the animal. Our conclusions are based on data obtained in five environments differing in size and spatial layout. 

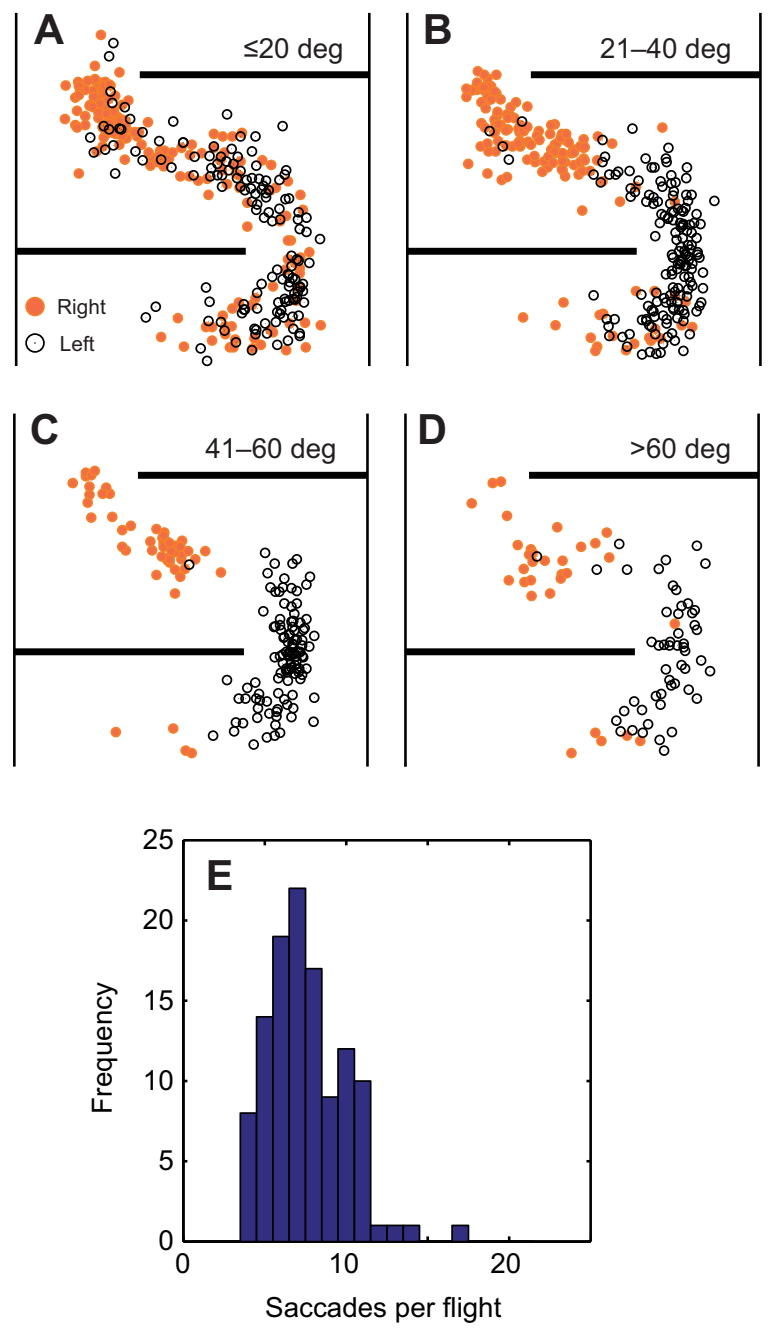

Fig. 9. (A-D) Location of saccades in the obstacle arena. Flight trajectories started at the bottom of figures. Saccades were classified according to their amplitude: (A) $\leq 20 \mathrm{deg}$, (B) 21-40 deg, (C) 41-60 deg and (D) >60 deg. Saccades to the left (right) are indicated by black open (orange filled) circles. The number of left and right saccades in each class, respectively, are as follows: (A) 143, 172; (B) 158, 150; (C) 112, 55; (D) 52, 31. (E) Frequency of saccade numbers per flight $(N=14, n=115)$.

Rather than the lateral visual field, where the OF, at least during forward flight, tends to be strongest, $\mathrm{OF}$ in eye regions looking well in front of the fly is suggested to be used by both the saccade and the velocity controller.

In the following, we will discuss how selected flight parameters are related to the visual environment and how they might be controlled by the experienced OF.

\section{Control of translational velocity}

Car drivers normally decelerate when obstacles restrict their path and blowflies also decelerate when obstacles come closer. This phenomenon has also been described for bees flying in flight tunnels of changing width (Baird et al., 2010; Portelli et al., 2011; Srinivasan et al., 1996). Interestingly, no differences in average flight velocity were found in Drosophila cruising in cylindrical flight arenas of considerably different diameters $[1.0 \mathrm{~m}$ (Tammero and Dickinson, 2002a); 0.14m (Mronz and Lehmann, 2008)], whereas David (David, 1982) found an increase of flight speed with increasing width
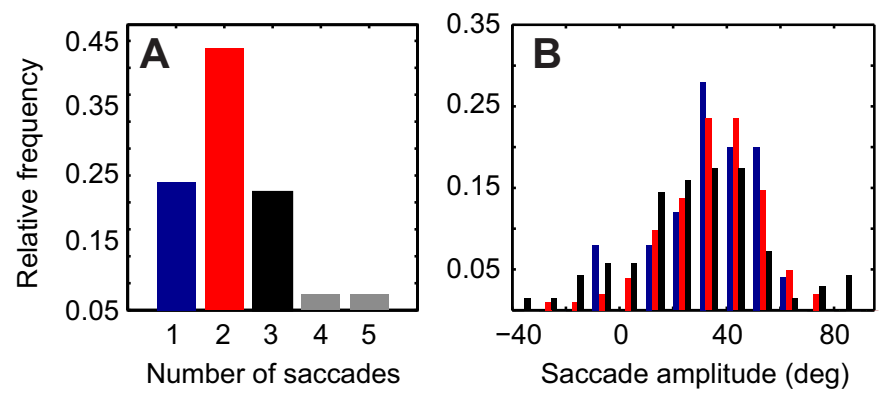

Fig. 10. (A) Relative frequency of saccade numbers per flight attributed to clearance of the first obstacle (see Fig.6A for the area within the obstacle tunnel considered). Only trajectories were taken into account where the body long-axis orientation deviated by no more than 20 deg from the tunnel long axis, and flight direction deviated by no more than 10 deg from the body long-axis orientation at the moment the fly entered the evaluation area. (B) Relative frequency of saccade amplitudes attributed to clearance of the first obstacle. Saccades either occurred in triplets (black) or doublets (red) performed in succession within the evaluation area during a given flight, or appeared in isolation (blue) $(N=14, n=105)$.

of horizontal wind tunnels. This apparent discrepancy might be the consequence of largely different spatial constraints in tunnel-like versus cylindrical flight arenas.

The most plausible sensory cue controlling flight velocity is the OF generated during flight [tethered flight (e.g. Götz, 1968; Theobald et al., 2010); free flight (Baird et al., 2005; Baird et al., 2006; Baird et al., 2010; David, 1979; David, 1982; Dyhr and Higgins, 2010; Farina et al., 1995; Fry et al., 2009; Frye and Dickinson, 2007; Kern and Varjú, 1998; Preiss, 1993; Serres et al., 2008; Straw et al., 2010)]. It appears that by adjusting their flight speed, insects keep the OF on their eyes at a 'preset' total strength (Srinivasan et al., 1996). Accordingly, insects decelerate when the translational OF increases, for instance, while passing a narrow gap or flying in a narrow tunnel, as has been observed in bees (Baird et al., 2010; Portelli et al., 2011; Srinivasan et al., 1991; Srinivasan et al., 1996), moths (Verspui and Gray, 2009) and blowflies (present study).

Our results indicate that not all parts of the visual field of the flies contribute to the input of the velocity controller. This suggestion was based on the assumption that those eye regions across which the overall OF is kept largely constant in different environments may be decisive in controlling the animal's translation velocity. We were able to determine the OF across different eye regions on the basis of behavioral data obtained in five flight environments, which differed largely in size and spatial layout. Although this selection of environments inevitably comprises only a small range of potential environments in which blowflies show flight behavior, the spatial constraints set by the tested environment go along with a wide range of systematic environment-dependent variations of flight parameters, such as flight velocity.

Whereas the OF in the most lateral and ventral parts of the visual field depends considerably on the environmental context, in the moderately ventral (from the horizon to $40 \mathrm{deg}$ beneath) and frontolateral visual field the $\mathrm{OF}$ is kept largely constant irrespective of the flight arena. Therefore, we suggest that the latter eye region is most relevant for velocity control of blowflies. Frontolaterally located eye regions have also been demonstrated to play a major role in velocity control in bumblebees (Baird et al., 2010). Recently, Portelli et al. (Portelli et al., 2011) concluded that honeybees also adapt their flight velocity to keep the retinal velocity relatively constant in the 

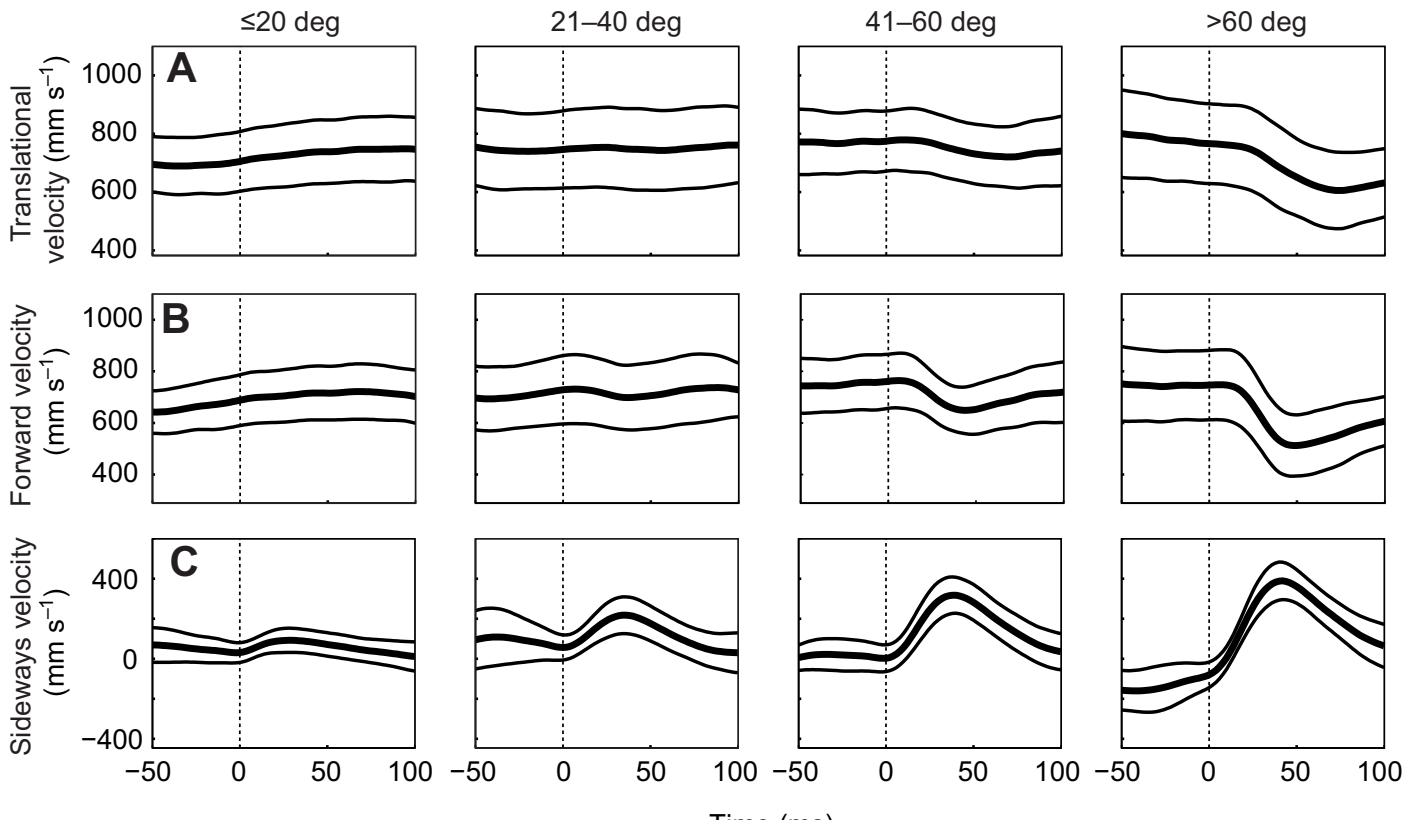

Fig. 11. Saccade-triggered time course of (A) total translation velocity and its (B) forward and (C) sideways components. Range of saccade amplitudes: $\leq 20 \mathrm{deg}(n=237), 21-40 \mathrm{deg}(n=258), 41-60 \mathrm{deg}(n=149)$ and $>60 \mathrm{deg}(n=76)$. The dashed vertical line (0 ms) denotes the beginning of saccades (threshold: $\left.200 \mathrm{deg} \mathrm{s}^{-1}\right)$. Data are means \pm s.d.

frontolateral visual field, independent of the spatial layout of the environment. In line with our results, viewing directions of $45 \mathrm{deg}$ in the bee's visual field yielded a less variable OF than viewing directions of $90 \mathrm{deg}$ in flight tunnels varying in width along their horizontal and/or vertical extent. These findings do not contradict studies on honeybees (e.g. Baird et al., 2006) or on Drosophila (e.g. David, 1979; Fry et al., 2009), where flight speed could be influenced by the OF caused by floor and/or side wall structures. The patterns employed in these studies also stimulated those eye regions that we concluded were involved in speed control for blowflies, but also for bees (Baird et al., 2010; Portelli et al., 2011).

The OF has been used by us as a first approximation of the sensory input of the velocity controller, although the computations in the visual motion pathway do not lead to a true representation of velocity, but also depend on texture and contrast of the environment. Moreover, spatial integration of local motion measurements across the visual field is nonlinear (for reviews, see Egelhaaf, 2006; Egelhaaf and Borst, 1993). Hence, future analysis should test whether our conclusions concerning the eye regions involved in velocity control are still valid if the specific features of biological motion computation are taken into account. Moreover, similar to those free-flight studies mentioned above on bees and Drosophila, all our conclusions on velocity control of blowflies are, inevitably, only correlative because they are based on free-flight behavior in a range of environments. Further analyses, potentially on tethered flying animals, where the stimuli to which the animal is exposed can be varied more systematically than is possible in free flight, are relevant to further challenge these conclusions on velocity control.

\section{Control of saccadic turns}

Our experiments revealed that in blowflies, in addition to their direction, the amplitude and frequency of saccades are the most relevant parameters to adjust turning behavior in response to environmental conditions; in contrast, saccade duration stays rather constant. How saccades of Drosophila depend on the environment can be inferred from two studies based on cylindrical flight arenas with largely different diameters: $1.0 \mathrm{~m}$ (Tammero and Dickinson, 2002a) and $0.14 \mathrm{~m}$ (Mronz and Lehmann, 2008). Mean saccade amplitudes and peak velocities were found to be considerably larger in the small flight arena than in the large one, reflecting the necessity to execute very sharp turns under spatial constraints to avoid collisions with the arena walls. Our finding that saccade amplitude - and with it saccade peak velocity - increases with decreasing timeto-contact is in agreement with this observation. In contrast to our results on blowflies, the duration of Drosophila free-flight saccades was concluded to depend on the flight arena and to increase notably with saccade amplitude (Mronz and Lehmann, 2008). The reasons for this difference are not clear, but may be species specific.

There is consensus that OF plays a decisive role in controlling the direction and amplitude of saccades. However, it is still inconclusive which OF parameters may be decisive. The times at which saccades are elicited in Drosophila and their direction were concluded to depend on the characteristics of retinal image expansion (Tammero and Dickinson, 2002a). In tethered flying Drosophila, saccades could be elicited in most parts of the visual field by expansion stimuli, with reduced response probabilities in frontal and rear regions and a rather broad probability peak in the lateral visual field (Bender and Dickinson, 2006; Tammero and Dickinson, 2002b; Tammero et al., 2004). On this basis it was concluded that image expansion in the front triggers landing and lateral expansion primarily collision avoidance responses. Hence, collision avoidance is assumed to be most effective during flight with a strong sideways component (Tammero et al., 2004). This conclusion has recently been challenged by correlating the occurrence of saccades of freeflying Drosophila with predictions based on hypothetical expansionand rotation-sensitive control systems (Mronz and Lehmann, 2008). A significantly higher correlation was found assuming a rotationsensitive system. These results led to the suggestion that image expansion on the side helps to achieve centering, for instance, in flight tunnels. 

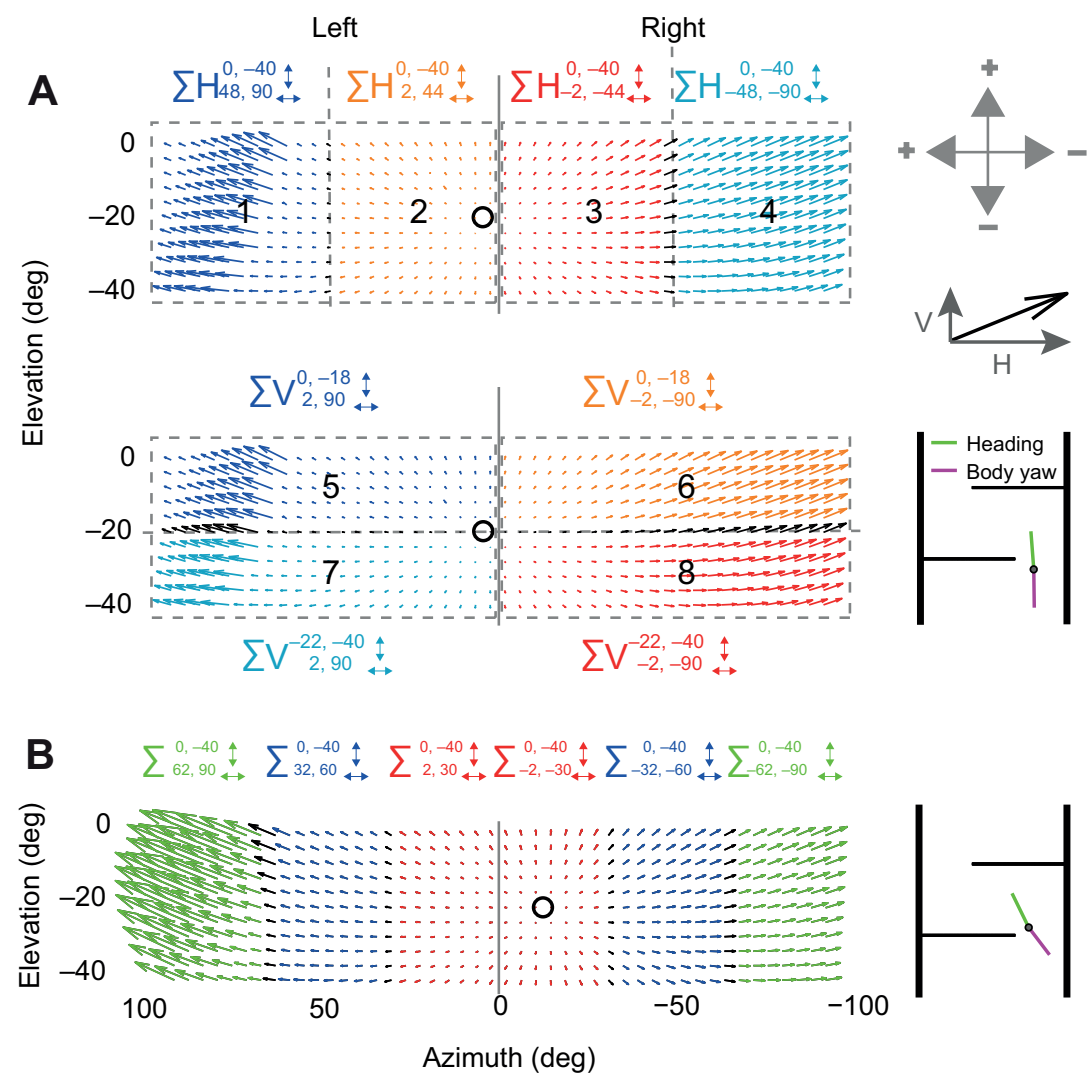

Fig. 12. Regions of the intersaccadic optic flow (OF) fields used to calculate the image expansion measure and example OF fields corresponding to two different flight sections of a single trajectory in the obstacle tunnel (see diagrams to the right). Each section corresponds to the time step from 26 to $24 \mathrm{~ms}$ prior to the next saccade. Both saccades are to the left. Saccade amplitudes were $40 \mathrm{deg}(\mathrm{A})$ and $30 \mathrm{deg}(\mathrm{B})$. For illustration purposes, the spatial resolution in the figure is 4 deg only; the calculations are based on a sample raster of $2 \times 2$ deg. The location of the focus of expansion (FOE) is indicated by a black circle. (A) To calculate a measure of expansion congruency of OF, four areas of equal width were defined, grouped about the frontal midline (1-4, top; differently colored arrows; black arrows highlight borders between regions). In addition - separately for either side - the flow field was divided into its upper and lower halves (5-8, bottom; differently colored arrows). Image expansion was approximated separately for the left and right side assuming the FOE was at \pm 46 deg in azimuth. The FOEs were at 0 deg elevation with respect to the horizon, i.e. -20 deg with respect to the eye because the head was pitch upwards by $20 \mathrm{deg}$. Horizontal image expansions were calculated as the difference of summed signed (top right diagram) horizontal flow components ( $\mathrm{H}$, see insets) between regions 1 and 2 (left) and regions 3 and 4 (right). Vertical expansions were calculated as the difference of summed signed vertical flow components (V) between regions 5 and 7 (left) and regions 6 and 8 (right). Horizontal and vertical expansions on either side were summed to yield the total expansion, i.e. our measure of expansion congruency. Diagram top right: front-to-back flow on the left (right) eye has a positive (negative) sign. Upwards (downwards) flow has a positive (negative) sign. (B) To calculate the difference in OF strength, six areas of equal width were defined, grouped about the frontal midline (differently colored arrows; black arrows highlight borders between regions). The lengths of all vectors within a given area were summed, yielding a measure of OF strength. The sign of the difference of OF strengths within areas located symmetrically with respect to the frontal midline (vectors of same color) was used as predictor of saccade direction.

Our results suggest that blowflies do not rely on the retinal location of the FOE for eliciting saccades. Rather, we can predict to some extent the direction of saccades from the difference of expansion congruency in $\mathrm{OF}$ as well as from the total amount of OF on either side of the visual field during the previous ISI. The spatial pooling of local motion information across large parts of the visual field, as is the basis of both OF measures, might be accomplished by individual or groups of motion-sensitive lobula plate tangential cells (LPTCs) (for reviews, see Borst et al., 2010; Egelhaaf, 2006). Indeed, the differential output of two model LPTCs, sensitive to horizontal image motion, located in both halves of the visual system of a behavioral blowfly model (CyberFly) is appropriate to some extent to control saccade generation with the goal of avoiding collisions with the walls of a flight arena (Lindemann et al., 2008). Also expansion-based mechanisms of saccade control do not necessarily require neurons that are sensitive to image expansion; rather, such a mechanism could rely on the signals of a combination of LPTCs with different preferred directions. Very recently, de Vries and Clandinin (de Vries and Clandinin, 2012) identified three cell types in the lobula complex of Drosophila that are selectively sensitive to looming stimuli and mimic objects on a direct collision course. Because unilaterally presented looming stimuli trigger saccadic turns in flying Drosophila (Tammero and Dickinson, 2002a; Tammero and Dickinson, 2002b), such neurons - if they exist in blowflies as well - might be involved in the saccadic steering behavior characterized in our study.

Our results suggest that the lateral parts of the visual field are not involved in determining saccade direction in blowflies. This feature might be related to the way blowflies fly: during ISIs they predominantly fly forward, with some sideways component after saccades that shifts the FOE slightly towards frontolateral locations (Fig. 13D). Because the sideways movements increase with saccade size and saccades, on average, are largest in the cubic arena, only in the latter environment FOE locations exceeding $50 \mathrm{deg}$ occur, 

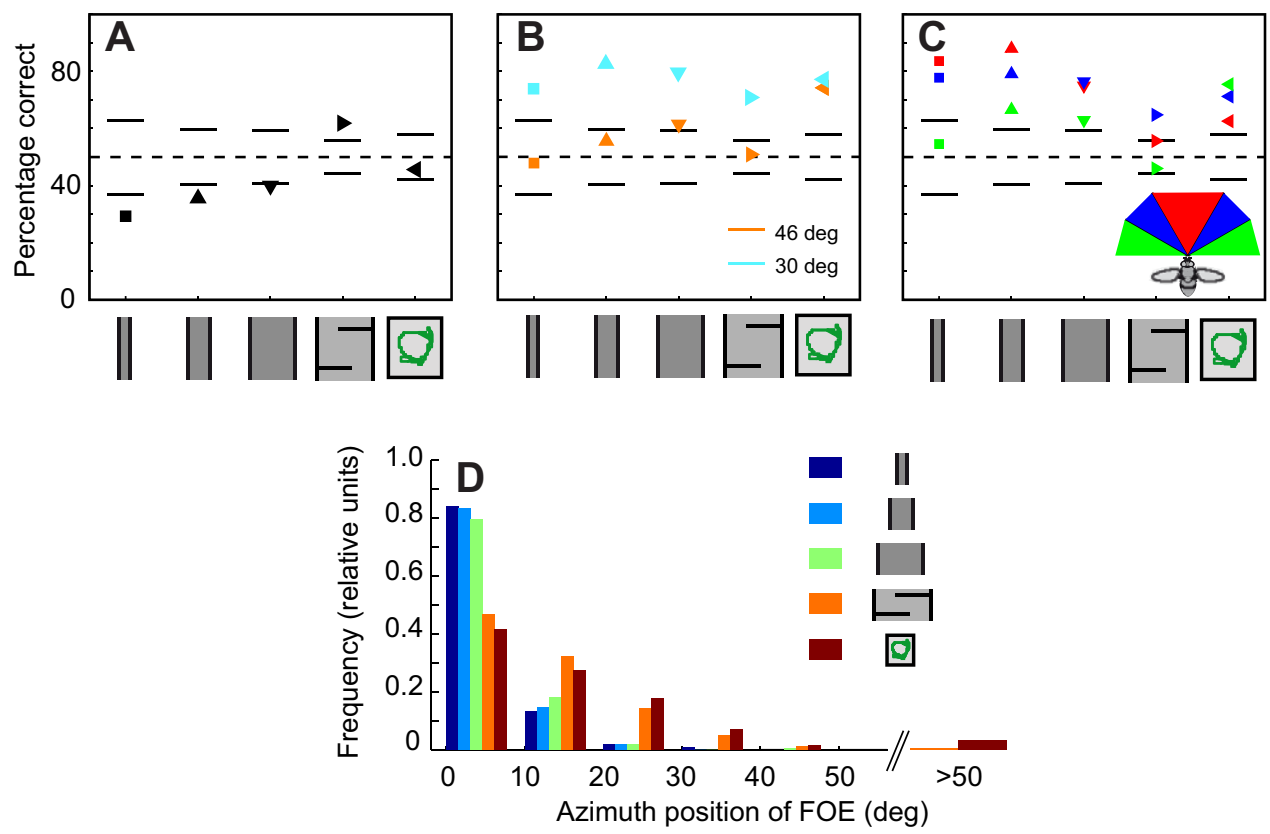

Fig. 13. (A) Percentage of correct predictions of saccade direction based on the FOE bearing in the OF field (OF calculated from 20 deg above to -20 deg below the horizon; azimuth from -90 deg to +90 deg; 0 deg is the frontal midline). The next saccade was predicted to be in the direction opposite to the FOE side. (B) Percentage of correct predictions based on the difference of the expansion congruency of OF in two symmetrical heterolateral eye regions with a vertical extent of $40 \mathrm{deg}$ centered about the horizon; in azimuth regions were 60 or 90 deg wide with an FOE at 30 or 46 deg, respectively (see Fig. $12 \mathrm{~A}$ ). (C) Percentage of correct predictions based on the difference of the total OF from selected heterolateral eye regions (elevation: from 20 deg above to -20 deg below horizon; azimuth: see inset and Fig. 12B). The next saccade was predicted to be in the direction of the side with smaller total OF. Black horizontal lines in A-C depict the 99\% confidence limits for binomial distributions (Sokal and Rohlf, 1981; Rohlf and Sokal, 1981). Predictions were made for a $10 \mathrm{~ms}$ ISI between 30 and $20 \mathrm{~ms}$ prior the next saccade. For $A-C$, the number of flies $(N)$ and of trajectories $(n)$ are as follows: narrow, $N=5, n=102$; medium, $N=11, n=178$; wide, $N=11, n=199$; obstacle tunnel, $N=14, n=535$; and cubic arena (van Hateren and Schilstra, 1999 ), $N=5, n=265$. (D) Relative frequencies of FOE bearing in the five different flight arenas. For OF calculations, head pitch was fixed at 20 deg upwards. Numbers on the $x$-axis indicate the edges used for binning.

though only at a very low frequency (Fig. 13D). Because the FOE is located only rarely beyond $30 \mathrm{deg}$ in the lateral visual field under all tested spatial conditions, the prediction of saccade direction on the basis of the expansion congruency of OF may be much better for FOEs assumed at $\pm 30 \mathrm{deg}$ rather than $\pm 45 \mathrm{deg}$ (Fig. 13B). In contrast, in Drosophila - which are able to hover and fly sideways (e.g. Ristroph et al., 2009) - lateral and even rear portions of the visual field have also been shown to be involved in saccade control. Therefore, a mechanism eliciting saccades in Drosophila that favors image expansion with an FOE at more lateral retinal positions than in Calliphora is plausible from a functional point of view, as has been concluded on the basis of free-flight studies (Tammero and Dickinson, 2002a), but also in tethered flight (Bender and Dickinson, 2006; Tammero and Dickinson, 2002b; Tammero et al., 2004).

Predicting saccade direction correctly with a probability of approximately $80 \%$ is far from being perfect. However, the mechanisms proposed here are still very simplistic and do not take into account the well-established non-linearities of the motion detection system (for reviews, see Borst et al., 2010; Egelhaaf, 2009). Moreover, saccade control might be influenced not only by visual input but also by other factors not controlled for during our experiments. In fruit flies, frequent 'spontaneous' saccades not triggered by visual cues (Bender and Dickinson, 2006) have been interpreted as part of a systematic search strategy, for instance, to localize attractive odors (Reynolds and Frye, 2007). Based on this finding, it is possible that a fraction of the saccades observed in our tunnels is actively generated to probe the three-dimensional layout of the environment based on the saccade-accompanying sideways movements. Depth can be judged best based on the OF generated by translational movements performed perpendicular to the direction of interest. Hence, the sideways motion component after saccades may facilitate the extraction of depth information in the frontolateral visual field. Sideways motion plays an important role in the context of peering movements in locusts and praying mantids (Kral et al., 2000; Sobel, 1990). Accordingly, the lateral movements of bees often generated close to objects have been attributed to an active vision strategy that facilitates the judgment of distances to objects (see Boeddeker and Hemmi, 2010) and to solve navigation tasks (Dittmar et al., 2010; Braun et al., 2012).

Body saccades tend to occur with some regularity in both blowflies (see Schilstra and van Hateren, 1999) and Drosophila (Tammero and Dickinson, 2002a), which suggests that saccades are triggered by an internal clock. This idea is not supported by our experimental evidence, which showed that saccade frequency varied between the different environments (see also Tammero and Dickinson, 2002a; Dickinson, 2005). Moreover, a behavioral blowfly model generates regular saccades without explicit modeling an internal clock for saccade generation (Lindemann et al., 2008).

\section{Saccades and translational velocity}

In blowflies, saccade rate in a given environment only slightly increases with translation velocity. This is similar in Drosophila (Mronz and Lehmann, 2008). However, the saccade rate of blowflies distinctly depends on the three-dimensional layout of the environment - being by far larger in the obstacle tunnel than in the straight tunnels. In the straight tunnels, the $\mathrm{OF}$ in frontolateral eye 
regions results from more distant structures, given that flies tend to be oriented along the tunnels' long axis. Hence, at a given flight velocity, frontolateral $\mathrm{OF}$ is weaker in the straight tunnels than in the obstacle tunnel, which may account for the lower saccade rate. Flies fly faster and need to change flight direction to avoid colliding with obstacles less frequently in open terrain. Accordingly, saccade rate is, on average, lower and saccade amplitudes are smaller the longer the estimated time-to-contact to an obstacle. Neurons, signaling time-to-contact either of an approaching object or during the animal's approach towards surfaces, have been described in various animals, including frogs (Nakagawa and Kang, 2010), gerbils (Shankar and Ellard, 2000), pigeons (Frost and Sun, 2004), hawk moths (Wicklein and Strausfeld, 2000), mantids (Yamawaki and Toh, 2009), locusts (Rind and Simmons, 1997; Gabbiani et al., 1999) and Drosophila (de Vries and Clandinin, 2012; Fotowat et al., 2009). In blowflies, such neurons have not yet been characterized.

The sideways movement components after saccades probably have their origin in inertial forces because the sideways velocity after saccades increases with saccade amplitude. Nonetheless, flies may actively control sideways movement, although Drosophila and hoverflies appear to do this to a much larger extent than blowflies (Schilstra and van Hateren, 1999; Ristroph et al., 2009; Sugiura and Dickinson, 2009; Braun et al., 2010; Geurten et al., 2010). Partial drift compensation presumably results from the fact that blowfly saccades are a combination of yaw, roll and pitch movements, leading to a banking behavior like that of airplanes during sharp turns (Schilstra and van Hateren, 1999; van Hateren and Schilstra, 1999; Braun et al., 2010). The sideways motion components of Calliphora just after saccades have been suggested to have functional significance. Previous studies have shown that motion-sensitive LPTCs probably involved in flight control directly encode information about intersaccadic sideways motion of the animal, and thus indirectly about the spatial layout of the environment (Karmeier et al., 2006; Kern et al., 2005; Kern et al., 2006; van Hateren et al., 2005). In addition, a saccadic controller as part of a model of blowfly visual course control (CyberFly) with sideways drift was shown to be slightly superior to a controller without sideways drift, because it is thought to facilitate the CyberFly to extract distance information in the frontolateral visual field (Lindemann et al., 2008).

The temporal relationship between saccades and a corresponding decrease in flight velocity differs for Drosophila and blowflies. Drosophila's peak saccade velocity tends to coincide with the drop in flight velocity (Mronz and Lehmann, 2008; Tammero and Dickinson, 2002a). The difference may be due to the fact that Drosophila saccades were not resolved on the basis of body orientation, but as a change in flight direction. As a consequence of inertia, these changes may occur slightly later than changes in body orientation (Schilstra and van Hateren, 1999). Trajectory-based saccade characterization is likely to lead to considerably larger saccade durations in Drosophila [exceeding $100 \mathrm{~ms}$ (Mronz and Lehmann, 2008; Tammero and Dickinson, 2002a)] than described for blowflies (approximately $30 \mathrm{~ms}$; Fig. 6F, Fig. 11) (Schilstra and van Hateren, 1999). If body yaw is determined and used for saccade analysis - as in the present paper - the saccade duration of Drosophila drops down to the $30 \mathrm{~ms}$ range (Bergou et al., 2010; Fry et al., 2003). Head saccades in blowflies are even shorter than body saccades (Schilstra and van Hateren, 1999; van Hateren and Schilstra, 1999), indicating that the head can turn even more rapidly than the body, which prolongs the ISIs that we here suggest to be used by blowflies for controlling saccades and translation velocity.

\section{NOTE ADDED IN PROOF}

Since acceptance of this paper another study dealing with fly free-flight behaviour has been published (van Breugel and Dickinson, 2012). One focus of this study is collision avoidance behaviour in Drosophila and, in this context, how saccadic turns are elicited. These issues overlap to some extent with problems addressed in the present paper.

\section{ACKNOWLEDGEMENTS}

A portion of the experiments was conducted by undergraduate students and by Frederique Oddos. We gratefully acknowledge the assistance of Grit Schwerdtfeger. Claudius Strub wrote the software to calculate optic flow.

\section{FUNDING}

The study was supported by the Deutsche Forschungsgemeinschaft (DFG).

\section{REFERENCES}

Baird, E., Srinivasan, M. V., Zhang, S. and Cowling, A. (2005). Visual control of flight speed in honeybees. J. Exp. Biol. 208, 3895-3905.

Baird, E., Srinivasan, M. V., Zhang, S., Lamont, R. and Cowling, A. (2006). Visual control of flight speed and height in the honeybee. In: From Animals to Animats 9, SAB 2006 (ed. S. Nolfi, G. Baldassare, R. Calabretta, J. Hallam, D. Marocco, O. Miglino, J.-A. Meyer and D. Parisi), pp. 40-51. Berlin: Springer.

Baird, E., Kornfeldt, T. and Dacke, M. (2010). Minimum viewing angle for visually guided ground speed control in bumblebees. J. Exp. Biol. 213, 1625-1632.

Bender, J. A. and Dickinson, M. H. (2006). Visual stimulation of saccades in magnetically tethered Drosophila. J. Exp. Biol. 209, 3170-3182.

Bergou, A. J., Ristroph, L., Guckenheimer, J., Cohen, I. and Wang, Z. J. (2010). Fruit flies modulate passive wing pitching to generate in-flight turns. Phys. Rev. Lett 104, 148101.

Boeddeker, N. and Hemmi, J. M. (2010). Visual gaze control during peering flight manoeuvres in honeybees. Proc. Biol. Sci. 277, 1209-1217.

Boeddeker, N., Kern, R. and Egelhaaf, M. (2003). Chasing a dummy target: smooth pursuit and velocity control in male blowflies. Proc. Biol. Sci. 270, 393-399.

Boeddeker, N., Lindemann, J. P., Egelhaaf, M. and Zeil, J. (2005). Responses of blowfly motion-sensitive neurons to reconstructed optic flow along outdoor flight paths. J. Comp. Physiol. A 191, 1143-1155.

Boeddeker, N., Dittmar, L., Stürzl, W. and Egelhaaf, M. (2010). The fine structure of honeybee head and body yaw movements in a homing task. Proc. Biol. Sci. 277, 1899-1906.

Borst, A., Haag, J. and Reiff, D. F. (2010). Fly motion vision. Annu. Rev. Neurosci. 33, 49-70.

Braun, E., Geurten, B. and Egelhaaf, M. (2010). Identifying prototypical components in behaviour using clustering algorithms. PLOS ONE 5, e9361.

Braun, E., Dittmar, L., Boeddeker, N. and Egelhaaf, M. (2012). Prototypical components of honeybee homing flight behavior depend on the visual appearance of objects surrounding the goal. Front. Behav. Neurosci. 6, 1.

Budick, S. A. and Dickinson, M. H. (2006). Free-flight responses of Drosophila melanogaster to attractive odors. J. Exp. Biol. 209, 3001-3017.

Budick, S. A., Reiser, M. B. and Dickinson, M. H. (2007). The role of visual and mechanosensory cues in structuring forward flight in Drosophila melanogaster. J. Exp. Biol. 210, 4092-4103.

Cardé, R. T. and Willis, M. A. (2008). Navigational strategies used by insects to find distant, wind-borne sources of odor. J. Chem. Ecol. 34, 854-866.

Collett, T. S. (2002). Insect vision: controlling actions through optic flow. Curr. Biol. 12, R615-R617.

Collett, T. S. and Land, M. F. (1975). Visual control of flight behaviour in the hoverfly Syritta pipiens L. J. Comp. Physiol. A 99, 1-66.

David, C. T. (1979). Optomotor control of speed and height by free-flying Drosophila. J. Exp. Biol. 82, 389-392.

David, C. T. (1982). Compensation for height in the control of groundspeed by Drosophila in a new, 'barber's pole' wind tunnel. J. Comp. Physiol. A 147, 485-493.

de Vries, S. E. J. and Clandinin, T. R. (2012). Loom-sensitive neurons link computation to action in the Drosophila visual system. Curr. Biol. 22, 353-362.

Dickinson, M. H. (2005). The initiation and control of rapid flight maneuvers in fruit flies. Integr. Comp. Biol. 45, 274-281.

Dittmar, L., Stürzl, W., Baird, E., Boeddeker, N. and Egelhaaf, M. (2010). Goal seeking in honeybees: matching of optic flow snapshots? J. Exp. Biol. 213, 29132923.

Dyhr, J. P. and Higgins, C. M. (2010). The spatial frequency tuning of optic-flowdependent behaviors in the bumblebee Bombus impatiens. J. Exp. Biol. 213, 1643 1650.

Egelhaaf, M. (2006). The neural computation of visual motion. In Invertebrate Vision (ed. E. Warrant and D.-E. Nilsson), pp. 399-461. Cambridge: Cambridge University Press.

Egelhaaf, M. (2009). Insect motion vision. Scholarpedia 4, 1671

Egelhaaf, M. and Borst, A. (1993). Movement detection in arthropods. In Visual Motion and its Role in the Stabilization of Gaze (ed. F. A. Miles and J. Wallman), pp. 53-77. Amsterdam: Elsevier.

Farina, W. M., Kramer, D. and Varjú, D. (1995). The response of the hovering hawk moth Macroglossum stellatarum to translatory pattern motion. J. Comp. Physiol. A 176, 551-562.

Foley, J. D., van Dam, A., Feiner, S. K. and Hughes, J. F. (1996). Computer Graphics: Principles and Practice, 2nd edn. Boston, MA: Addison Wesley.

Fotowat, H., Fayyazuddin, A., Bellen, H. J. and Gabbiani, F. (2009). A novel neuronal pathway for visually guided escape in Drosophila melanogaster. J. Neurophysiol. 102, 875-885. 
Frost, B. J. and Sun, H. (2004). The biological basis of time-to-collision computation. In Advances in Psychology 135 - Time-to-Contact (ed. H. Hecht and G. J. P. Savelsbergh), pp. 13-37. Amsterdam: Elsevier.

Fry, S. N., Sayaman, R. and Dickinson, M. H. (2003). The aerodynamics of free-flight maneuvers in Drosophila. Science 300, 495-498.

Fry, S. N., Rohrseitz, N., Straw, A. D. and Dickinson, M. H. (2009). Visual control of flight speed in Drosophila melanogaster. J. Exp. Biol. 212, 1120-1130.

Frye, M. A. and Dickinson, M. H. (2007). Visual edge orientation shapes free-flight behavior in Drosophila. Fly 1, 153-154

Gabbiani, F., Krapp, H. G. and Laurent, G. (1999). Computation of object approach by a wide-field, motion-sensitive neuron. J. Neurosci. 19, 1122-1141.

Geurten, B. R. H., Kern, R., Braun, E. and Egelhaaf, M. (2010). A syntax of hoverfly flight prototypes. J. Exp. Biol. 213, 2461-2475.

Gibson, J. J. (1979). The Ecological Approach to Visual Perception. Boston, MA Houghton Mifflin.

Götz, K. G. (1968). Flight control in Drosophila by visual perception of motion. Biol. Cybern. 4, 199-208.

Hesselberg, T. and Lehmann, F. O. (2009). The role of experience in flight behaviour of Drosophila. J. Exp. Biol. 212, 3377-3386.

Karmeier, K., van Hateren, J. H., Kern, R. and Egelhaaf, M. (2006). Encoding of naturalistic optic flow by a population of blowfly motion-sensitive neurons. $J$. Neurophysiol. 96, 1602-1614.

Kern, R. and Varjú, D. (1998). Visual position stabilization in the hummingbird hawk moth, Macroglossum stellatarum L. I. Behavioural analysis. J. Comp. Physiol. A 182 225-237.

Kern, R., van Hateren, J. H., Michaelis, C., Lindemann, J. P. and Egelhaaf, M. (2005). Function of a fly motion-sensitive neuron matches eye movements during free flight. PLOS Biol. 3, e171.

Kern, R., van Hateren, J. H. and Egelhaaf, M. (2006). Representation of behaviourally relevant information by blowfly motion-sensitive visual interneurons requires precise compensatory head movements. J. Exp. Biol. 209, 1251-1260.

Kirchner, W. H. and Srinivasan, M. V. (1989). Freely flying honeybees use image motion to estimate object distance. Naturwissenschaften 76, 281-282.

Koenderink, J. J. (1986). Optic flow. Vision Res. 26, 161-179.

Koenderink, J. J. and van Doorn, A. J. (1987). Facts on optic flow. Biol. Cybern. 56 247-254.

Kral, K., Vernik, M. and Devetak, D. (2000). The visually controlled prey-capture behaviour of the European mantispid Mantispa styriaca. J. Exp. Biol. 203, 2117-2123.

Lappe, M. (2000). Neuronal Processing of Optic Flow. San Diego, CA: Academic Press.

Lindemann, J. P., Boeddeker, N. and Egelhaaf, M. (2003). 3D-reconstruction of insect flight trajectories from 2D image sequences. In Proceedings of the 5th Meeting of the German Neuroscience Society (ed. N. Elsner and H. Zimmermann), 1065pp. Stuttgart: Thieme.

Lindemann, J. P., Weiss, H., Möller, R. and Egelhaaf, M. (2008). Saccadic flight strategy facilitates collision avoidance: closed-loop performance of a cyberfly. Biol. Cybern. 98, 213-227.

McArthur, K. L. and Dickman, J. D. (2011). State-dependent sensorimotor processing: gaze and posture stability during simulated flight in birds. $J$ Neurophysiol. 105, 1689-1700.

Menzel, R. (2009). Working memory in bees: also in flies? J. Neurogenet. 23, 92-99.

Mronz, M. and Lehmann, F. O. (2008). The free-flight response of Drosophila to motion of the visual environment. J. Exp. Biol. 211, 2026-2045.

Nakagawa, H. and Kang, H. (2010). Collision-sensitive neurons in the optic tectum of the bullfrog, Rana catesbeiana. J. Neurophysiol. 104, 2487-2499.

Portelli, G., Ruffier, F., Roubieu, F. L. and Franceschini, N. (2011). Honeybees' speed depends on dorsal as well as lateral, ventral and frontal optic flows. PLoS ONE 6, e19486.

Preiss, R. (1993). Visual control of orientation during swarming flight of desert locusts In Sensory Systems of Arthropods (ed. K. Wiese), pp. 273-287. Basel: Birkhäuse Verlag.

Reiser, M. B. and Dickinson, M. H. (2010). Drosophila fly straight by fixating objects in the face of expanding optic flow. J. Exp. Biol. 213, 1771-1781.

Reynolds, A. M. and Frye, M. A. (2007). Free-flight odor tracking in Drosophila is consistent with an optimal intermittent scale-free search. PLOS ONE 2, e354.
Ribak, G. and Swallow, J. G. (2007). Free flight maneuvers of stalk-eyed flies: do eye-stalks affect aerial turning behavior? J. Comp. Physiol. A 193, 1065-1079.

Rind, F. C. and Simmons, P. J. (1997). Signaling of object approach by the DCMD neuron of the locust. J. Neurophysiol. 77, 1029-1033.

Ristroph, L., Berman, G. J., Bergou, A. J., Wang, Z. J. and Cohen, I. (2009) Automated hull reconstruction motion tracking (HRMT) applied to sideways maneuvers of free-flying insects. J. Exp. Biol. 212, 1324-1335.

Rohlf, F. J. and Sokal, R. R. (1981). Statistical Tables, 2nd edn. New York: Freeman

Schilstra, C. and van Hateren, J. H. (1999). Blowfly flight and optic flow. I. Thorax kinematics and flight dynamics. J. Exp. Biol. 202, 1481-1490.

Serres, J. R., Masson, G. P., Ruffier, F. and Franceschini, N. (2008). A bee in the corridor: centering and wall-following. Naturwissenschaften 95, 1181-1187.

Shankar, S. and Ellard, C. (2000). Visually guided locomotion and computation of time-to-collision in the Mongolian gerbil (Meriones unguiculatus): the effects of frontal and visual cortical lesions. Behav. Brain Res. 108, 21-37.

Sobel, E. C. (1990). The locust's use of motion parallax to measure distance. J. Comp. Physiol. A 167, 579-588.

Sokal, R. R. and Rohlf, F. J. (1981). Biometry, 2nd edn. New York: Freeman.

Srinivasan, M. V., Lehrer, M., Kirchner, W. H. and Zhang, S. W. (1991). Range perception through apparent image speed in freely flying honeybees. Vis. Neurosci. 6, 519-535.

Srinivasan, M. V., Zhang, S. W., Lehrer, M. and Collett, T. S. (1996). Honeybee navigation en route to the goal: visual flight control and odometry. J. Exp. Biol. 199, 237-244.

Straw, A. D., Lee, S. and Dickinson, M. H. (2010). Visual control of altitude in flying Drosophila. Curr. Biol. 20, 1550-1556.

Sugiura, H. and Dickinson, M. H. (2009). The generation of forces and moments during visual-evoked steering maneuvers in flying Drosophila. PLOS ONE 4, e4883.

Tammero, L. F. and Dickinson, M. H. (2002a). The influence of visual landscape on the free flight behavior of the fruit fly Drosophila melanogaster. J. Exp. Biol. 205, 327-343

Tammero, L. F. and Dickinson, M. H. (2002b). Collision-avoidance and landing responses are mediated by separate pathways in the fruit fly, Drosophila melanogaster. J. Exp. Biol. 205, 2785-2798.

Tammero, L. F., Frye, M. A. and Dickinson, M. H. (2004). Spatial organization of visuomotor reflexes in Drosophila. J. Exp. Biol. 207, 113-122.

Theobald, J. C., Ringach, D. L. and Frye, M. A. (2010). Dynamics of optomotor responses in Drosophila to perturbations in optic flow. J. Exp. Biol. 213, 1366-1375. van Breugel, F. and Dickinson, M. H. (2012). The visual control of landing and obstacle avoidance in the fruit fly Drosophila melanogaster. J. Exp. Biol. 215, 17831798.

van Hateren, J. H. and Schilstra, C. (1999). Blowfly flight and optic flow. II. Head movements during flight. J. Exp. Biol. 202, 1491-1500.

van Hateren, J. H., Kern, R., Schwerdtfeger, G. and Egelhaaf, M. (2005). Function and coding in the blowfly $\mathrm{H} 1$ neuron during naturalistic optic flow. J. Neurosci. 25 , 4343-4352.

Verspui, R. and Gray, J. R. (2009). Visual stimuli induced by self-motion and objectmotion modify odour-guided flight of male moths (Manduca sexta L.). J. Exp. Biol. 212, 3272-3282.

Wagner, H. (1985). Aspects of the free flight behaviour of houseflies (Musca domestica). In Insect Locomotion (ed. M. Gewecke and G. Wendler), pp. 223-232. Berlin: Paul Parey Verlag.

Wagner, H. (1986). Flight performance and visual control of flight of the free-flying housefly (Musca domestica). I. Organization of the flight motor. Philos. Trans. R. Soc. Lond. B 312, 527-551.

Wicklein, M. and Strausfeld, N. J. (2000). Organization and significance of neurons that detect change of visual depth in the hawk moth Manduca sexta. J. Comp. Neurol. 424, 356-376.

Yamawaki, Y. and Toh, Y. (2009). Responses of descending neurons to looming stimuli in the praying mantis Tenodera aridifolia. J. Comp. Physiol. A 195, 253-264.

Zeil, J. (1983). Sexual dimorphism in the visual system of flies: The free flight behaviour of male Bibionidae (Diptera). J. Comp. Physiol. A 150, 395-412.

Zeil, J. (1986). The territorial flight of male houseflies (Fannia canicularis). Behav. Ecol. Sociobiol. 19, 213-219. 